\title{
Viscoelasticity of vesicle dispersions
}

\author{
Kazuhiko Seki $^{\mathrm{a}, 1}$, Shigeyuki Komura ${ }^{\mathrm{b}, 2}$ \\ a Department of Energy Sciences, Faculty of Science and Engineering, Tokyo Institute of Technology, \\ Meguro-ku. Tokyo 152, Japan

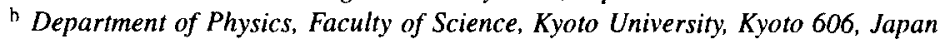

Received 30 June 1994; revised 27 January 1995

\begin{abstract}
Linear viscoelastic properties of dispersions of vesicles and microemulsions are investigated in detail. Each droplet is considered to be coated by a film of amphiphilic molecules (fluid membrane) whose shape is mainly governed by the bending rigidity rather than the surface tension. In addition to this elastic property, we also take into account the membrane compressibility which plays an important role in the macroscopic viscoelastic behavior of the system. For dilute emulsions, we calculated the effective complex viscosity by generalizing Taylor's old calculation. The mechanical force balance condition at the membrane can be expressed by using the generalized Laplace's formula derived for compressible fluid membranes. On the basis of the cell model, we also calculated the effective complex viscosity for nondilute emulsions. Comparing various characteristic time scales both for vesicles and microemulsions, we discuss the detectable relaxation modes in the viscoelastic experiments.
\end{abstract}

\section{Introduction}

Properties of flexible fluid membranes made of amphiphilic molecules have attracted great interest in connection with various fields such as the statistical physics, quantum field theory, physical chemistry or biophysics [1]. Such membranes are typically realized in spherically closed membranes of lipid bilayers called "vesicles" which are exemplified by red blood cells. This type of thin-walled fluid vesicles have received great attention as models of cell membranes. Shape transformation among various conformations can be

\footnotetext{
'Address after 1 April 1994: Theoretical Chemistry Lab., National Institute of Materials and Chemical Research, Tsukuba 305, Japan. e-mail address: ck938@nimc.go.jp

2 e-mail address: komuraøton. scphys .kyoto-u.ac.jp
} 
caused by changing, e.g., the osmotic conditions, the temperature or the composition of the lipids [2]. These properties of vesicles might be closely related to the physiological functions of biomembranes. Similar properties are also realized in a microemulsion system being homogeneous mixtures of oil, water and surfactants [3]. In both cases, amphiphilic molecules orient their polar heads towards water and their aliphatic tails away from it, decreasing the surface tension drastically to the level of practically zero. Instead of the surface tension, it is widely recognized that the deformation of the membrane is mainly governed by the elastic bending energy [4]. Since the associated bending rigidity is known to be of the order of $k_{B} T$, membranes can easily fluctuate due to thermal agitations. Although microemulsion droplets differ from vesicles by several decades in length scale (see Section 6), the ruling physics behind it is expected to be similar.

Such sheets of membranes have two important features. First, a typical size of a vesicle formed by bilayers is in the $\mu \mathrm{m}$ range while its thickness is in the $\mathrm{nm}$ range so that the bilayer can be regarded as a two-dimensional system embedded in a three-dimensional space. Second, an ordinary membrane is in a fluid state at room temperature and it is supposed to have no in-plane shear resistivity. The curvature model was originally proposed for such a fluid membrane according to a phenomenological consideration [4].

In order to make our understanding of the bending energy and associated hydrodynamics deeper, not only static measurements but also dynamical measurements of the membrane system are quite important. In fact, there have been several dynamical measurements of vesicles (fluorescence microscopy [5], optical video-microscopy [612], reflection interference contrast microscopy [13-15]) and microemulsions (neutron scattering [16-18], dynamical Kerr effect [19]). By knowing the relaxation time of a small deformation of a vesicle or a microemulsion droplet, one can quantitatively determine the bending rigidity. Along with these experiments, several people calculated the time correlation function of the out-of-plane displacement for a spherically closed fluid membrane. The first work by Shneider, Jenkins and Webb [5] was generalized by Milner and Safran to the case of non-zero spontaneous curvature [20]. The important assumption in their calculations is that the total area is a conserved quantity as well as the total volume both for vesicles and microemulsion droplets. In order to incorporate these two constraints simultaneously, they introduced the idea of "constant excess area" together with the unknown Lagrange multiplier. On the other hand, Van der Linden, Bedeaux and Borkovec insisted that only the area should be kept constant for vesicles, whereas only the volume constraint is necessary for microemulsion droplets since the supply and the loss of surfactants from the bulk phase would take place in a short enough time scale compared to the deformation of droplets [21]. Van der Linden et al. or Komura and Seki [22] obtained different dynamical correlation functions from the previous result by assuming that only the total volume should be kept constant. Some people calculated the relaxation time upon taking into account the compressibility of the membrane [23-27].

Recently, Smeulders, Blom and Mellema performed a viscoelastic measurement on an emulsion of relatively small vesicles, $r_{0} \simeq 40-100 \mathrm{~nm}[28-30]$. A distinct feature 
of their observation is that there exist two relaxation processes; one related to the translational ordering of the vesicles that are subjected to a shear flow, and the other, appearing at higher frequencies, has been attributed to the membrane deformation. From the characteristic time of the latter relaxation process, they determined several elastic constants and viscosities of the membrane as well as the bending rigidity by changing the size of the droplets. Their experimental data has been analyzed according to the theoretical prediction by Oldroyd with a modified relaxation time including the bending rigidity $[31,32]$. One of their interesting findings is the deformation mode dependent on the shear modulus, although Helfrich assumed vanishing of it in his original model [4]. Recently, viscosity of the $L_{3}$-phase in amphiphilic systems has been also reported $[33,34]$.

Up to now there has been no complete calculation of the effective viscosity for an emulsion of fluid membranes governed by the bending energy except for some special cases. In the present article, we consider the viscoelasticity of an emulsion of vesicles or a microemulsion system. Since the viscosity can be regarded as an example of a response function, its calculation is also interesting from the point of view of statistical mechanics. We take into account the membrane compressibility which plays an important role in the viscoelastic behavior. In order to grasp the physical properties of the membrane, we calculated the effective complex viscosity by generalizing Taylor's old calculation $[35,36]$. The hydrodynamical boundary condition at the membrane represents the balance of force at the membrane [22], and we employ the generalized Laplace formula derived for compressible fluid membranes [22,37,38]. The spirit of our calculation is similar to the recent work by Onuki [26]. In addition to the dilute case, we also derived the complex viscosity for nondilute emulsions on the basis of the cell model.

In fact, the calculation of the effective viscosity of dispersed systems has a very long history. The first hydrodynamic calculation was given by Einstein for suspensions of solid spheres in the steady shear flow [39]. Taylor extended Einstein's approach to the liquid droplets in the continuous aqueous phase [35]. Both of the results were limited to the dilute case. Later Frölich and Sack [40] and Oldroyd [31] argued the viscoelasticity of suspensions and emulsions, respectively. For nondilute dispersions, extensive calculations have been performed by several people by using the cell model $[41,42]$.

The outline of this article is as follows. In Section 2, after some mathematical definitions, we give a description of the compressible fluid membrane and the mechanical boundary condition at the interface. In Section 3, the constitutive equation and formal expression of the effective viscosity are presented. The viscoelasticity is calculated for dilute and nondilute dispersions in Sections 4 and 5, respectively. In the last section, comparing various characteristic time scales for vesicles and microemulsions, we discuss the detectable relaxation modes in the viscoelastic experiments. 


\section{Closed fluid membranes}

\subsection{Preparations}

First, we will collect some formulas from differential geometry. One can, in general, parameterize a two-dimensional membrane in a three-dimensional space by two real inner coordinates $s=\left(s^{1}, s^{2}\right)$. The shape of the membrane is then described by a threedimensional vector $r=r(s)$. At each point on the membrane, there are two tangent vectors $\boldsymbol{r}_{i}=\partial \boldsymbol{r} / \partial s^{i}$ with $i=1,2$. The outward unit normal vector $n$ is perpendicular to these tangent vectors, i.e., $n=\left(\boldsymbol{r}_{1} \times \boldsymbol{r}_{2}\right) /\left|\boldsymbol{r}_{1} \times \boldsymbol{r}_{2}\right|$.

All properties related to the intrinsic geometry of the membrane are expressed in terms of the metric tensor defined by the inner product of the tangential vectors

$$
g_{i j}=\boldsymbol{r}_{i} \cdot \boldsymbol{r}_{j}
$$

Two important quantities are the determinant and the inverse of the metric tensor which will be denoted by

$$
g=\operatorname{det}\left(g_{i j}\right) \text { and } g^{i j}=\left(g_{i j}\right)^{-1} .
$$

In addition, one has to consider the (extrinsic) curvature tensor given by

$$
h_{i j}=\boldsymbol{n} \cdot \boldsymbol{r}_{i j}=-\boldsymbol{n}_{\boldsymbol{i}} \cdot \boldsymbol{r}_{j}
$$

where $\boldsymbol{r}_{i j}=\partial^{2} \boldsymbol{r} / \partial s^{i} \partial s^{j}$ and $\boldsymbol{n}_{i}=\partial \boldsymbol{n} / \partial s^{i}$. The third expression follows from the partial derivative of $n \cdot r_{j}=0$. Similar to Eq. (2.2), the determinant and the inverse of the curvature tensor are denoted by

$$
h=\operatorname{det}\left(h_{i j}\right) \text { and } h^{i j}=\left(h_{i j}\right)^{-1} .
$$

The mean curvature $H$ and the Gaussian curvature $K$ are calculated according to

$$
H=\frac{1}{2} g^{i j} h_{i j}=-\frac{1}{2}\left(c_{1}+c_{2}\right),
$$

and

$$
K=h / g=c_{1} c_{2},
$$

respectively, where $c_{1}$ and $c_{2}$ are two principle curvatures.

The covariant derivative $D_{j}$ of $f^{i}$ and $f_{i}$ are

$$
D_{j} f^{i}=\partial_{j} f^{i}+\Gamma_{k j}^{i} f^{k} \text { and } D_{j} f_{i}=\partial_{j} f_{i}-\Gamma_{i j}^{k} f_{k},
$$

respectively, with the Christoffel symbols $\Gamma_{i j}^{k}$,

$$
\Gamma_{i j}^{k}=g^{k l} \boldsymbol{r}_{l} \cdot \boldsymbol{r}_{i j},
$$

and $\partial_{i} \equiv \partial / \partial s^{i}$.

The metric and the curvature tensors are determined by the vector $r(s)$ and consist of six independent functions since both tensors are symmetric. In order to solve the inverse 
problem, namely, to determine the function $r(s)$ from the fundamental tensors, one has to solve the following equations:

$$
\boldsymbol{n}_{i}=-h_{i}^{j} \boldsymbol{r}_{j}
$$

and

$$
\boldsymbol{r}_{i j}=\Gamma_{i j}^{k} \boldsymbol{r}_{k}+h_{i j} \boldsymbol{n}
$$

which are called Weingarten equation and Gaussian equation, respectively.

\subsection{Compressible fluid membranes}

Using the notations in the previous subsection, we consider an emulsion system where each dispersed droplet is considered to be coated by a fluid membrane consisting of amphiphilic molecules such as lipids or surfactants. The equilibrium shape of the fluid membrane is determined by the minimization of the following shape energy $[4,37]$ :

$$
H_{f}=\oint_{A_{0}} \sigma\left(\rho_{s}\right) \mathrm{d} A+\frac{1}{2} \kappa \oint_{A_{0}}\left(c_{1}+c_{2}-c_{0}\right)^{2} \mathrm{~d} A .
$$

In the above, $\mathrm{d} A=\sqrt{g} \mathrm{~d} s^{1} \mathrm{~d} s^{2}$ is the surface element while $A_{0}$ is the surface of a particular closed membrane over which the integrals are performed. (We use $\oint$ for the surface integral in order to distinguish from the volume integral $\int$.) Furthermore, $\sigma\left(\rho_{s}\right)$ is the bare surface tension dependent on the areal density $\rho_{s}$ of amphiphilic molecules on the membrane [43], $\kappa$ the bending rigidity and $c_{0}$ the spontaneous curvature.

It is important to mention that due to the incompressible assumption for the ambient fluids (see later Eq. (3.2)), the shape energy Eq. (2.11) should be minimized under the constraint of constant enclosed volume. This constraint is only a mathematical requirement in the present paper and one can also argue the case of compressible surrounding fluids [25]. Appropriate constraint for the real systems is a matter of discussion as has been stated in the introduction. In principle, one can also consider the situation in which $\kappa$ and/or $c_{0}$ depend also on $\rho_{s}$. However this effect is expected to be negligibly small compared to the bare surface tension [23] and we shall put these quantities as constants.

Exchange of molecules between the membrane and the ambient fluid takes place through desorption and adsorption. Desorption is a thermally activated process. The characteristic time for a molecule to remain in the membrane is proportional to $\exp (\Delta E /$ $k_{B} T$ ), where $\Delta E$ is the energy barrier per molecule associated with the desorption process. For a phospholipid in the bilayer membrane, the estimated typical sticking time (at room temperature) ranges from several hours to a couple of days, i.e., $\simeq 10^{5}[\mathrm{~s}]$, which provides a measure of the time scale within which a new chemical equilibrium can be attained. This estimation implies that such a relaxation process takes a rather long time in the case of bilayers made of lipids such as red blood cells. Hence one can assume that the number of molecules in the membrane remains almost constant during 
the time scale of the experiments which is much shorter than the sticking time $[44,45]$. Keeping these facts in mind, we assume throughout this paper that the total number of amphiphilic molecules $N$ is a conserved quantity. Here $N$ is given by the integral of $\rho_{s}$ over the surface of the membrane, i.e.,

$$
N=\oint_{A_{0}} \rho_{s} \mathrm{~d} A .
$$

By further assuming that the density fluctuation is induced due to the local areal change of the membrane, the variation of $\mathrm{Eq}$. (2.12) yields

$$
0=\delta \rho_{s} \mathrm{~d} A+\rho_{s} \delta \mathrm{d} A,
$$

which will be used in the following discussion.

Some comments are necessary for the above assumption. Typical sticking time scale for a single chain surfactant in micelles is much shorter compared to the lipids and is in the order of $10^{-5}-10^{-3}$ [s]. Hence for a supramolecular structure of surfactants, Eq. (2.13) holds only for a short time scale. One should note that there exists also an open system, such as black films, where the lipid bilayer exchanges molecules with the outer system. In this case, $N$ is no longer a constant while $\rho_{s}$ remains unchanged with respect to the small change in the total area [44].

By calculating the first variation of the shape energy Eq. (2.11) with respect to an infinitesimal displacement normal to the membrane under the constraint of fixed $N$, we obtain the following normal restoring force due to the membrane [5]:

$$
F_{\|}=2 \Sigma\left(\rho_{S}\right) H-\kappa\left(2 H+c_{0}\right)\left(2 H^{2}-2 K-c_{0} H\right)-2 \kappa \nabla_{L B}^{2} H,
$$

where $\Sigma\left(\rho_{s}\right)$ is the apparent surface tension defined by [43]

$$
\Sigma\left(\rho_{s}\right) \equiv \sigma\left(\rho_{s}\right)-\rho_{s} \frac{\partial \sigma\left(\rho_{s}\right)}{\partial \rho_{s}},
$$

and $\nabla_{L B}^{2}$ is the Laplace-Beltrami operator on the surface given by

$$
\nabla_{L B}^{2}=\frac{1}{\sqrt{g}} \partial_{i}\left(g^{i j} \sqrt{g} \partial_{j}\right) .
$$

In order to make this paper self-contained, we give a concise derivation of Eq. (2.14) in Appendix A. The second term on the r.h.s. of Eq. (2.15) represents the reduction of the surface tension.

It is important to notice that in the shape energy Eq. (2.11), the compressibility of the fluid membrane has been implicitly taken into account through the change of $\rho_{s}$. This can be seen as follows. Due to the fluctuation of the local density $\delta \rho_{s}$ of amphiphilic molecules around the equilibrium (or mean) value of $\rho_{s}$ denoted as $\rho_{s 0}$, the apparent surface tension $\mathrm{Eq}$. (2.15) can be different from point to point on the surface being expressed as $\Sigma\left(\rho_{s}\right)=\Sigma\left(\rho_{s 0}\right)+\delta \Sigma\left(\rho_{s}\right)$, where $[23,43,46,47]$

$$
\delta \Sigma\left(\rho_{s}\right)=-\frac{B}{\rho_{s 0}} \delta \rho_{s}, \quad \text { with } B=\rho_{s 0}^{2}\left(\frac{\partial^{2} \sigma\left(\rho_{s}\right)}{\partial \rho_{s}^{2}}\right)_{\rho_{s}=\rho_{s 0}} .
$$


In the above, $B$ is called the two-dimensional (in-plane) compression modulus and is put hereafter as a constant. The former relation in Eq. (2.17) can be also regarded as an equation of state of the membrane as a two-dimensional fluid [27].

It is known that the compressible nature of the membrane leads to a considerable effect on the hydrodynamical properties of the interface [52]. The change in the membrane shape due to the motion of the surrounding fluid is coupled to the change in $\rho_{s}$ which in turn affects the surface tension as seen by Eq. (2.17). If $\rho_{s}$ varies over the surface, the apparent surface tension Eq. (2.15) is not constant as well and this fact gives rise to the tangential restoring force due to the membrane such that [55]

$$
F_{\perp \alpha}=\operatorname{grad}_{\perp \alpha} \Sigma\left(\rho_{s}\right)
$$

where $\operatorname{grad}_{\perp \alpha}$ is the $\alpha$-component of the gradient operator on the surface given by

$$
\operatorname{grad}_{\perp \alpha}=\frac{\partial}{\partial x_{\alpha}}-n_{\alpha} n_{\beta} \frac{\partial}{\partial x_{\beta}},
$$

while $n_{\alpha}$ are components of the normal vector (which should not be confused with $\boldsymbol{n}_{i}=\partial \boldsymbol{n} / \partial s^{i}$ ). (We shall use Greek indices for the range $1,2,3$.)

In order to construct the boundary condition that must be satisfied at the interface between two viscous fluids in motion, we follow the discussion by Landau and Lifshitz [52]. According to Eqs. (2.14) and (2.18), the balance of force per unit membrane leads to

$$
\left(\Pi_{\alpha \beta}-\Pi_{\alpha \beta}^{\prime}\right) n_{\beta}+F_{\|} n_{\alpha}+F_{\perp \alpha}=0,
$$

or more explicitly

$$
\begin{aligned}
& \left(\Pi_{\alpha \beta}-\Pi_{\alpha \beta}^{\prime}\right) n_{\beta}+\operatorname{grad}_{\perp \alpha} \Sigma\left(\rho_{s}\right) \\
& \quad+\left[2 \Sigma\left(\rho_{s}\right) H-\kappa\left(2 H+c_{0}\right)\left(2 H^{2}-2 K-c_{0} H\right)-2 \kappa \nabla_{L B}^{2} H\right] n_{\alpha}=0 .
\end{aligned}
$$

where, as will be given later in Section $3.1, \Pi_{\alpha \beta}$ is the fluid stress tensor outside of the membrane and $\Pi_{\alpha \beta}^{\prime}$ is that inside of the membrane. Here and below we shall use the prime in order to distinguish the quantities of the fluid inside from the corresponding quantities of the fluid outside. For the direction normal to the membrane, Eq. (2.21) reduces to the well-known Laplace's formula when $\kappa=0$ and $\Sigma\left(\rho_{s}\right)$ represents a constant surface tension [52]. A more general case has been discussed by Onuki and Kawasaki $[38,43]$.

In addition to the equation of motion for the ambient fluids with the boundary condition Eq. (2.21), we need another equation since the variable $\rho_{s}$ has been introduced in our problem. This is an equation of continuity of amphiphilic molecules, expressing the conservation of the local number of molecules (Eq. (2.13)). According to the hydrodynamics of two-dimensional fluids $[27,38,53]$, this can be written in general as

$$
\frac{\partial \rho_{s}}{\partial t}+\operatorname{div}_{\perp}\left(\rho_{s} v_{s}\right)+\frac{\rho_{s}}{2 g} \frac{\partial g}{\partial t}=0
$$


See Eq. (2.2) for $g$ and $\boldsymbol{v}_{s}$ is the velocity of amphiphilic molecules composing the membrane and will be put equal to those of ambient fluids. (The allowance of the slippage between the membrane and the fluids was considered by Oldroyd [32] and recently by Onuki $[26,38]$.) The precise mathematical definition of the term $\operatorname{div}_{\perp}\left(\rho_{s} v_{s}\right)$ in the language of differential geometry is given in Appendix B. The last term in Eq. (2.22) simply expresses the density fluctuation due to the local areal change.

We finally comment that another way of incorporating the membrane compressibility is to add a compressional energy term such as

$$
H_{c}=\frac{1}{2} B \oint_{A_{0}}\left(\frac{\delta \rho_{s}}{\rho_{s 0}}\right)^{2} \mathrm{~d} A
$$

to Eq. (2.11) if the surface tension $\sigma\left(\rho_{s}\right)$ in the first term is put as a constant (zero) [48-51]. However, the compressibility is more generally taken into account in Eq. (2.11) than by Eq. (2.23).

\subsection{Spherically closed membranes}

For our later purpose, we summarize here several expressions related to a spherically closed fluid membrane of radius $r_{0}$ at zero temperature. By specifying the internal coordinates as $\left(s^{1}, s^{2}\right)=(\theta, \varphi)$, we introduce the following three unit vectors as a local basis:

$$
\boldsymbol{e}_{r}=\left(\begin{array}{c}
\sin \theta \cos \varphi \\
\sin \theta \sin \varphi \\
\cos \theta
\end{array}\right), \quad \boldsymbol{e}_{\theta}=\left(\begin{array}{c}
\cos \theta \cos \varphi \\
\cos \theta \sin \varphi \\
-\sin \theta
\end{array}\right), \quad \boldsymbol{e}_{\varphi}=\left(\begin{array}{c}
-\sin \varphi \\
\cos \varphi \\
0
\end{array}\right)
$$

With these notations, the undeformed reference state is described by

$$
R=r_{0} e_{r}
$$

Now we consider the membrane slightly distorted from the reference state. Any deformed state of the membrane without any overhangs can then be parameterized by using the normal vector $N$ in the reference state, i.e., $N=\left(R_{1} \times R_{2}\right) /\left|R_{1} \times R_{2}\right|$, in the following way:

$$
\boldsymbol{r}=\boldsymbol{R}+\ell(\theta, \varphi, t) N=\left[r_{0}+\ell(\theta, \varphi, t)\right] \boldsymbol{e}_{r} .
$$

Here the variable $\ell(\theta, \varphi, t)$ represents the transverse (out-of-plane) displacement field which can generally depend on time $t$. (Here we did not include the in-plane displacements which are irrelevant in the bending energy up to the second order in terms of displacement fields. In-plane displacements should be taken into account in describing polymerized elastic membranes.) A straightforward calculation up to first order in terms of the out-of-plane displacement $\ell$ yields the following expression for the normal vector:

$$
\boldsymbol{n} \approx \boldsymbol{e}_{r}-\frac{1}{r_{0}} \frac{\partial \ell}{\partial \theta} e_{\theta}-\frac{1}{r_{0} \sin \theta} \frac{\partial \ell}{\partial \varphi} \boldsymbol{e}_{\varphi}
$$


and for twice the mean curvature $H$ and the Gaussian curvature $K$ :

$$
2 H \approx-\frac{2}{r_{0}}+\frac{1}{r_{0}^{2}}\left(2+\nabla_{\perp}^{2}\right) \ell(\theta, \varphi, t),
$$

and

$$
K \approx \frac{1}{r_{0}^{2}}-\frac{1}{r_{0}^{3}}\left(2+\nabla_{\perp}^{2}\right) \ell(\theta, \varphi, t),
$$

respectively, where

$$
\nabla_{\perp}^{2}=\frac{1}{\sin \theta} \frac{\partial}{\partial \theta}\left(\sin \theta \frac{\partial}{\partial \theta}\right)+\frac{1}{\sin ^{2} \theta} \frac{\partial^{2}}{\partial \varphi^{2}} .
$$

The linearized equation of continuity can be written from Eq. (2.22) as [27]

$$
\frac{\partial}{\partial t} \delta \rho_{s} \approx-\rho_{s 0} \operatorname{div}_{\perp} v_{s}-\frac{2 \rho_{s 0}}{r_{0}} \frac{\partial \ell}{\partial t}
$$

where

$$
\operatorname{div}_{\perp} \boldsymbol{v}_{s}=\frac{1}{r_{0} \sin \theta}\left[\frac{\partial}{\partial \theta}\left(\sin \theta v_{s \theta}\right)+\frac{\partial}{\partial \varphi} v_{s \varphi}\right]
$$

is the usual two-dimensional divergence of $v_{s}$ in the spherical coordinate.

For our later calculations, it is convenient to expand the function $\ell(\theta, \varphi, t)$ in terms of the spherical harmonics $Y_{n m}(\theta, \varphi)$,

$$
\ell(\theta, \varphi, t)=\sum_{n, m}^{\prime} \ell_{n m}(t) Y_{n m}(\theta, \varphi)
$$

As usual, we have $\ell_{m m}^{*}(t)=(-1)^{m} \ell_{n,-m}(t)$ in order to ensure that the displacement field is real (the asterisk denotes the complex conjugate value) and the summation with a prime runs over $n=1,2, \ldots$ and $|m| \leq n$. The term for $(n, m)=(0,0)$ is excluded here due to the constraint of constant enclosed volume. (In our later notation, the summation without any prime includes the $(n, m)=(0,0)$ term.) Hereafter, the well-known relation

$$
\nabla_{\perp}^{2} Y_{n m}(\theta, \varphi)=-n(n+1) Y_{n m}(\theta, \varphi)
$$

will be used frequently.

\section{Effective viscosity of dispersions}

\subsection{Constitutive equation}

Dispersions of small spherical droplets can be regarded as a homogeneous fluid when we concern the phenomena that occur in a much larger length scale than the average 
size of the dispersed droplets. One example is the rheological behavior of dispersions where one asks the stress needed to cause a given bulk motion. On measuring the rheological property, a dispersion can be considered as a homogeneous fluid with an effective viscosity. Here we have addressed the term homogeneous in a statistical sense, since the exact position and motion of droplets may differ for different realizations of experiments even if the macroscopic conditions such as the boundary conditions are prepared in the same manner. Hence we may observe, in principle, the ensemble average of the fluid velocity $\langle\boldsymbol{v}\rangle$ instead of its exact value $\boldsymbol{v}$. However, the ensemble average can not be calculated directly and we assume the ergodicity property of the system, namely, the equality of the ensemble average and the volume average. Study of the effective viscosity of dispersions has a fairly long history and its theoretical basis has been well established. In this section, we shall briefly outline the basic theory by Batchelor who provided a general constitutive equation of suspensions [54] and extend it for emulsions.

First we consider an emulsion of fluid droplets being coated by a fluid membrane (discontinuous phase) and immersed in another fluid (continuous phase). As in the previous section, quantities with prime refer to those on the inside of the droplets while notations without prime correspond to those on the outside. Both fluids are assumed to be Newtonian in its stress behavior. Denoting the fluid velocity by $v^{(\prime)}$, the stress tensors are given by

$$
I_{\alpha \beta}^{\left({ }^{\prime}\right)}=-P^{\left({ }^{\prime}\right)} \delta_{\alpha \beta}+\sigma_{\alpha \beta}^{\left({ }^{\prime}\right)}=-P^{\left({ }^{\prime}\right)} \delta_{\alpha \beta}+\eta^{\left({ }^{\prime}\right)}\left(\frac{\partial v_{\alpha}^{\left({ }^{\prime}\right)}}{\partial x_{\beta}}+\frac{\partial v_{\beta}^{\left({ }^{\prime}\right)}}{\partial x_{\alpha}}\right),
$$

where primes with parentheses imply that the above equation holds both for inside and outside the membrane. In the last equation, $\eta^{\left({ }^{\prime}\right)}$ are the dynamic viscosities and we have also assumed that both fluids are incompressible, i.e.,

$$
\operatorname{div} \boldsymbol{v}^{\left({ }^{\prime}\right)}=0 .
$$

Notice that this condition leads to the constraint of constant enclosed volume.

The average of the stress tensor over the volume $V$ large enough to contain many droplets is

$$
\left\langle\Pi_{\alpha \beta}\right\rangle=\frac{1}{V} \int_{V} \Pi_{\alpha \beta} \mathrm{d} V
$$

This integral can be decomposed into the sum of the integrals over the volume of a typical droplet having the volume $V_{0}$ and the remaining part of the bulk $\bar{V}$. Then we have

$$
\left\langle\Pi_{\alpha \beta}\right\rangle=\frac{1}{V} \int_{\bar{V}}\left[-P \delta_{\alpha \beta}+\eta\left(\frac{\partial v_{\alpha}}{\partial x_{\beta}}+\frac{\partial v_{\beta}}{\partial x_{\alpha}}\right)\right] \mathrm{d} V+c \int_{V_{0}^{>}} \Pi_{\alpha \beta}^{\prime} \mathrm{d} V,
$$

where $c$ is the number of droplets per unit volume. Notice that $\bar{V}=V\left(1-c V_{0}\right)$ holds. Since we have assumed that $\bar{V}$ is completely occupied by the continuous phase (without 
prime), $V_{0}$ must include the interfacial layer. In order to stress that this boundary lies on the outer surface of the membrane, we use the notations $V_{0}^{>}$and $A_{0}^{>}$, which stand for the volume including the membrane and the membrane outer surface, respectively.

It is convenient to express $\mathrm{Eq}$. (3.4) in terms of surface integrals. For this purpose we employ the relations

$$
\left\langle\frac{\partial v_{\alpha}}{\partial x_{\beta}}\right\rangle \equiv \frac{1}{V} \int_{V} \frac{\partial v_{\alpha}}{\partial x_{\beta}} \mathrm{d} V=\frac{1}{V} \int_{\bar{V}} \frac{\partial v_{\alpha}}{\partial x_{\beta}} \mathrm{d} V+c \oint_{A_{0}^{>}} v_{\alpha} n_{\beta} \mathrm{d} A
$$

and

$$
\int_{V_{0}^{>}} \Pi_{\alpha \beta}^{\prime} \mathrm{d} V=\oint_{A_{0}^{>}} \Pi_{\alpha \gamma} x_{\beta} n_{\gamma} \mathrm{d} A-\int_{V_{0}^{>}} \frac{\partial \Pi_{\alpha \gamma}^{\prime}}{\partial x_{\gamma}} x_{\beta} \mathrm{d} V
$$

where the Gauss theorem has been applied in Eq. (3.5). Then Eq. (3.4) can be expressed as

$$
\begin{aligned}
\left\langle\Pi_{\alpha \beta}\right\rangle= & -\frac{1}{V} \int_{\bar{V}} P \delta_{\alpha \beta} \mathrm{d} V+\eta\left\langle\frac{\partial v_{\alpha}}{\partial x_{\beta}}+\frac{\partial v_{\beta}}{\partial x_{\alpha}}\right\rangle \\
& +c \oint_{A_{0}^{>}}\left[\Pi_{\alpha \gamma} x_{\beta} n_{\gamma}-\eta\left(v_{\alpha} n_{\beta}+v_{\beta} n_{\alpha}\right)\right] \mathrm{d} A-c \int_{V_{0}^{>}} \frac{\partial \Pi_{\alpha \gamma}^{\prime}}{\partial x_{\gamma}} x_{\beta} \mathrm{d} V .
\end{aligned}
$$

In general, the stress tensor can be divided into an isotropic part and an anisotropic part. The isotropic part is determined by the external conditions and is irrelevant to the argument of the constitutive equation. Hence we shall drop the first term on the r.h.s. of Eq. (3.7) and neglect hereafter the difference in the isotropic part of the stress tensor. Moreover the last term also vanishes since $\partial \Pi_{\alpha \gamma}^{\prime} / \partial x_{\gamma}=0$ within the droplet as long as the inertial forces can be neglected. The derived constitutive equation is, therefore, $[52,54]$

$$
\left\langle\Pi_{\alpha \beta}\right\rangle=\eta\left\langle\frac{\partial v_{\alpha}}{\partial x_{\beta}}+\frac{\partial v_{\beta}}{\partial x_{\alpha}}\right\rangle+c \oint_{A_{0}^{>}}\left[\Pi_{\alpha \gamma} x_{\beta} n_{\gamma}-\eta\left(v_{\alpha} n_{\beta}+v_{\beta} n_{\alpha}\right)\right] \mathrm{d} A
$$

Here the second term on the r.h.s. represents the extra stress due to the presence of the dispersed particles.

Although Eq. (3.8) has been first derived for a suspension of rigid particles dispersed in a Newtonian fluid [52,54], it is also applicable for emulsions where fluid droplets are immersed in another Newtonian fluid. To see this more clearly, we derive an alternative form of Eq. (3.8) [55]. If we denote the inner surface of the membrane by $A_{0}^{<}$, we have

$$
\oint_{A_{0}^{<}} n_{\alpha \gamma}^{\prime} x_{\beta} n_{\gamma} \mathrm{d} A=\oint_{A_{0}^{<}} \eta^{\prime}\left(v_{\alpha}^{\prime} n_{\beta}+v_{\beta}^{\prime} n_{\alpha}\right) \mathrm{d} A,
$$


where the isotropic pressure term has been dropped out. Using this equation and Eq. (3.8), we obtain

$$
\begin{aligned}
\left\langle\Pi_{\alpha \beta}\right\rangle=\eta & \left\langle\frac{\partial v_{\alpha}}{\partial x_{\beta}}+\frac{\partial v_{\beta}}{\partial x_{\alpha}}\right\rangle+c\left[\oint_{A_{0}^{>}} \Pi_{\alpha \gamma} x_{\beta} n_{\gamma} \mathrm{d} A-\int_{A_{0}^{<}} \Pi_{\alpha \gamma}^{\prime} x_{\beta} n_{\gamma} \mathrm{d} A\right] \\
& -c\left[\eta \oint_{A_{0}^{>}}\left(v_{\alpha} n_{\beta}+v_{\beta} n_{\alpha}\right) \mathrm{d} A-\eta^{\prime} \oint_{A_{0}^{<}}\left(v_{\alpha}^{\prime} n_{\beta}+v_{\beta}^{\prime} n_{\alpha}\right) \mathrm{d} A\right] .
\end{aligned}
$$

Taking the limit of the membrane thickness going to infinitely small and employing the "stick" boundary condition (continuity of the velocity) at the interface, we have [55]

$$
\begin{aligned}
\left\langle\Pi_{\alpha \beta}\right\rangle= & \eta\left\langle\frac{\partial v_{\alpha}}{\partial x_{\beta}}+\frac{\partial v_{\beta}}{\partial x_{\alpha}}\right\rangle+c \oint_{A_{0}^{>}}\left(\Pi_{\alpha \gamma}-\Pi_{\alpha \gamma}^{\prime}\right) x_{\beta} n_{\gamma} \mathrm{d} A \\
& -c\left(\eta-\eta^{\prime}\right) \oint_{A_{0}^{>}}\left(v_{\alpha} n_{\beta}+v_{\beta} n_{\alpha}\right) \mathrm{d} A .
\end{aligned}
$$

In the above, the stress difference $\left(\Pi_{\alpha \gamma}-\Pi_{\alpha \gamma}^{\prime}\right) x_{\beta} n_{\gamma}$ can be written in terms of the surface tension and the bending rigidity $[43,56]$.

In case there is no force associated with the interface, i.e. $\Pi_{\alpha \gamma}=\Pi_{\alpha \gamma}^{\prime}$, Eq. (3.11) reduces to

$$
\left\langle\Pi_{\alpha \beta}\right\rangle=\eta\left\langle\frac{\partial v_{\alpha}}{\partial x_{\beta}}+\frac{\partial v_{\beta}}{\partial x_{\alpha}}\right\rangle-c\left(\eta-\eta^{\prime}\right) \oint_{A_{0}^{>}}\left(v_{\alpha} n_{\beta}+v_{\beta} n_{\alpha}\right) \mathrm{d} A .
$$

Here the change in the viscosity due to the presence of droplets is proportional to the difference of the viscosities between the inside and outside of the droplet. Detailed discussions are left in Sections 4 and 5.

\subsection{Formal calculation}

In this subsection, we shall give a formal calculation of the effective viscosity on the basis of Eq. (3.8) under an imposed shearing motion. The flow field around a spherical droplet is assumed to be the "creeping flow" which satisfies the stationary Stokes equation $[5,20,21,58]$ :

$$
\eta^{\left({ }^{\prime}\right)} \nabla^{2} v^{\left({ }^{\prime}\right)}=\operatorname{grad} P^{\left({ }^{\prime}\right)}
$$

and simultaneously the incompressibility condition E4. ) , The solution to Eqs. (3.2) and (3.13) in spherical coordinates is expressed in terms of three scalar functions: $\psi(\boldsymbol{r}, t), \mathcal{X}(\boldsymbol{r}, t)$ and $P(\boldsymbol{r}, t)$ where $\psi$ and $\chi$ give solutions to the homogeneous equation 
$\nabla^{2} v=0$. Since all of these functions satisfy the Laplace equation (obviously $\nabla^{2} P=0$ ), they can be expanded in terms of solid spherical harmonics:

$$
\begin{aligned}
& \psi^{\prime}(\boldsymbol{r}, t)=\sum_{n, m}^{\prime} \psi_{n m}^{\prime}(t)\left(\frac{r}{r_{0}}\right)^{n} Y_{n m}(\theta, \varphi), \\
& \chi^{\prime}(\boldsymbol{r}, t)=\sum_{n, m}^{\prime} \chi_{n m}^{\prime}(t)\left(\frac{r}{r_{0}}\right)^{n} Y_{n m}(\theta, \varphi), \\
& P^{\prime}(r, t)=\sum_{n, m} P_{n m}^{\prime}(t)\left(\frac{r}{r_{0}}\right)^{n} Y_{n m}(\theta, \varphi),
\end{aligned}
$$

and

$$
\begin{aligned}
& \psi(\boldsymbol{r}, t)=\sum_{n, m}^{\prime} \psi_{n m}(t)\left(\frac{r_{0}}{r}\right)^{n+1} Y_{n m}(\theta, \varphi), \\
& \chi(r, t)=\sum_{n, m}^{\prime} \chi_{n m}(t)\left(\frac{r_{0}}{r}\right)^{n+1} Y_{n m}(\theta, \varphi), \\
& P(r, t)=\sum_{n, m} P_{n m}(t)\left(\frac{r_{0}}{r}\right)^{n+1} Y_{n m}(\theta, \varphi),
\end{aligned}
$$

describing the inside and outside of the fluid, respectively. For the pressure fields $P^{\prime}$ and $P$, we included $(n, m)=(0,0)$ mode (without prime in the summation) because of the existence of the finite non-fluctuating hydrostatic pressure which satisfies

$$
\left(P_{00}-P_{00}^{\prime}\right) r_{0}^{3}+2 \Sigma\left(\rho_{s 0}\right) r_{0}^{2}+\kappa c_{0} r_{0}\left(c_{0} r_{0}-2\right)=0
$$

This relation is called the "capillarity condition" $[21,22]$, and comes from the requirement that the undeformed reference sphere Eq. (2.25) is always a solution of the equilibrium shape equation [37]:

$$
P-P^{\prime}=F_{\|}
$$

According to the general solution of the Stokes equation given by Lamb [57], the velocity field on the inside of the droplet is given by [58]

$$
\begin{aligned}
\boldsymbol{v}^{\prime}(\boldsymbol{r}, t)= & \sum_{n, m}^{\prime}\left(\psi_{n m}^{\prime}(t) \operatorname{grad}+\chi_{n m}^{\prime}(t) \operatorname{rot} \boldsymbol{r}+\frac{(n+3)}{2 \eta^{\prime}(n+1)(2 n+3)} P_{n m}^{\prime}(t) r^{2} \mathrm{grad}\right. \\
& \left.-\frac{n}{\eta^{\prime}(n+1)(2 n+3)} P_{n m}^{\prime}(t) r\right)\left(\frac{r}{r_{0}}\right)^{n} Y_{n m}(\theta, \varphi)
\end{aligned}
$$

while the corresponding solution for outside the droplet is 


$$
\begin{aligned}
\boldsymbol{v}(\boldsymbol{r}, t)-\boldsymbol{v}^{\infty}(\boldsymbol{r}, t)= & \sum_{n, m}^{\prime}\left(\psi_{n m}(t) \operatorname{grad}+\chi_{n m}(t) \operatorname{rot} \boldsymbol{r}-\frac{(n-2)}{2 \eta n(2 n-1)} P_{n m}(t) r^{2} \operatorname{grad}\right. \\
& \left.+\frac{(n+1)}{\eta n(2 n-1)} P_{n m}(t) \boldsymbol{r}\right)\left(\frac{r_{0}}{r}\right)^{(n+1)} Y_{n m}(\theta, \varphi),
\end{aligned}
$$

where $v^{\infty}(r, t)$ is the unperturbed flow given by the boundary condition at infinite distance from the droplet given later in Eq. (3.24). In Eqs. (3.22) and (3.23), both the gradient and rotation operators act on the solid spherical harmonics outside the large bracket as well. Lamb showed that the radial component of the velocity involves $\psi$ and $P$ while $r \cdot \operatorname{rot} v$ is only a function of $\chi[57,58]$ and the terms involving $\chi$ always separate out during the calculation. Hence, as far as our present purpose is concerned, we can ignore $\chi^{\left({ }^{\prime}\right)}$ without loss of generality and this simplifies the problem to some extent $[5,20,21,58]$.

At large distances from the droplet, the velocity field is described by a linear velocity distribution

$$
v_{\alpha}^{\infty}(r, t)=p_{\alpha \beta}(t) x_{\beta},
$$

where $p_{\alpha \beta}(t)$ is a second-order symmetric tensor with $p_{\alpha \alpha}=0$ since the fluids are incompressible. Although $p_{\alpha \beta}(t)$ can vary with time arbitrarily, we shall consider only an oscillatory motion in the form of $e^{i \omega t}$ with which all the time dependence will be taken into account hereafter. Following the symmetry argument on $p_{\alpha \beta}$ by Oldroyd [31], it suffices to consider only a pure elongational flow represented by

$$
p_{\alpha \beta}(t)=\left(\begin{array}{ccc}
-\Gamma & 0 & 0 \\
0 & -\Gamma & 0 \\
0 & 0 & 2 \Gamma
\end{array}\right) e^{i \omega t},
$$

in Cartesian coordinates. In spherical coordinates, Eq. (3.24) becomes

$$
v^{\infty}(r, t)=\Gamma \operatorname{grad}\left[r^{2} Y_{20}(\theta, \varphi)\right] e^{i \omega t},
$$

with

$$
\operatorname{grad}=e_{r} \frac{\partial}{\partial r}+e_{\theta} \frac{1}{r} \frac{\partial}{\partial \theta}+e_{\varphi} \frac{1}{r \sin \theta} \frac{\partial}{\partial \varphi} .
$$

The unknown coefficients in Eqs. (3.22) and (3.23) are determined according to the boundary conditions at $r=r_{0}$ and it turns out that only $\boldsymbol{P}_{20}$ contributes to the calculation of the effective viscosity. To the lowest order (in the absence of droplets), the first term in Eq. (3.8) is given by [59]

$$
\eta\left\langle\frac{\partial v_{\alpha}}{\partial x_{\beta}}+\frac{\partial v_{\beta}}{\partial x_{\alpha}}\right\rangle=\eta\left(\frac{\partial v_{\alpha}^{\infty}}{\partial x_{\beta}}+\frac{\partial v_{\beta}^{\infty}}{\partial x_{\alpha}}\right) \equiv \sigma_{\alpha \beta}^{\infty} .
$$

By using the following abbreviation for the surface integral in Eq. (3.8):

$$
J_{\alpha \beta} \equiv c \oint_{A_{0}^{>}}\left[I_{\alpha \gamma} x_{\beta} n_{\gamma}-\eta\left(v_{\alpha} n_{\beta}+v_{\beta} n_{\alpha}\right)\right] \mathrm{d} A,
$$


we show in Appendix $C$ that $[42,59]$

$$
J_{\alpha \beta}=-\frac{P_{20}}{4 \eta \Gamma} \phi \sigma_{\alpha \beta}^{\infty},
$$

where $\phi=(4 \pi / 3) c r_{0}^{3}$ is the volume fraction of the total droplets. Notice that $J_{\alpha \beta}$ vanishes in the absence of the droplets.

Since the effective viscosity $\eta^{*}$ is defined as

$$
\left\langle\Pi_{\alpha \beta}\right\rangle=\eta^{*}\left\langle\frac{\partial v_{\alpha}}{\partial x_{\beta}}+\frac{\partial v_{\beta}}{\partial x_{\alpha}}\right\rangle,
$$

it can be formally expressed as

$$
\eta^{*}=\eta\left(1-\frac{P_{20}}{4 \eta \Gamma} \phi\right),
$$

from Eqs. (3.28) and (3.30). We see that the knowledge of $P_{20} / \Gamma$ is sufficient to determine the effective viscosity.

In the present paper, we restrict ourselves to the case that the deformation of the membrane is small enough. A qualitative picture of the deformation process can be easily obtained. When the droplet of radius $r_{0}$ is deformed according to a shear flow of magnitude $\Gamma$, stress tending to elongate the droplet is of order $\eta \Gamma$. In the case of the vanishing surface tension, opposing $\eta \Gamma$ is a stress of magnitude $\kappa / r_{0}^{3}$, which tends to maintain the droplet in a spherical shape. Thus, the shape of the droplet in a steady shear flow is governed by a non-dimensional parameter, $\eta \Gamma r_{0}^{3} / \kappa$, with which the condition of small deformation can be expressed as $\eta \Gamma r_{0}^{3} / \kappa \ll 1$. The above dimensionless quantity is analogous to the capillary number $\eta \Gamma r_{0} / \Sigma$ of the system where the surface tension plays an important roll.

\section{Dilute case}

In this section, we calculate the effective viscosity of a dilute dispersion. To achieve this, we have to determine $P_{20}$ in Eq. (3.32) subjected to the appropriate boundary conditions. For such, we employ the "stick" boundary condition as has been argued in the foregoing sections and was used by several authors before [21,22,27]. This condition requires that the velocity of the molecules composing the membrane and the fluid velocity on both sides of the membrane are equal. Hence from Eq. (2.26), the condition is written as

$$
v=v^{\prime}=v_{s}=\frac{\partial r}{\partial t}
$$

at the surface. Since the velocity field is linear in the fluctuating amplitude $\ell$, one can impose the boundary conditions at $r=r_{0}$. The first set of boundary conditions comes from the continuity of the velocity. In the $\boldsymbol{e}_{\boldsymbol{r}}$-direction, this is written as 


$$
\begin{aligned}
& v_{r}\left(r=r_{0}\right)=\frac{1}{r_{0}} \sum_{n, m}^{\prime}\left(n \psi_{n m}^{\prime}+\frac{n}{2 \eta^{\prime}(2 n+3)} r_{0}^{2} P_{n m}^{\prime}\right) Y_{n m}(\theta, \varphi) e^{i \omega t} \\
& =-\frac{1}{r_{0}} \sum_{n, m}^{\prime}\left((n+1) \psi_{n m}-\frac{(n+1)}{2 \eta(2 n-1)} r_{0}^{2} P_{n m}\right) Y_{n m}(\theta, \varphi) e^{i \omega t}+v_{r}^{\infty}\left(r=r_{0}\right) \\
& =\sum_{n, m}^{\prime} i \omega \ell_{n m} Y_{n m}(\theta, \varphi) e^{i \omega t},
\end{aligned}
$$

where the last equation has been obtained by taking the time derivative of Eq. (2.33). Likewise, the continuity of the velocity in the $\boldsymbol{e}_{\theta}$-direction leads to

$$
\begin{aligned}
v_{\theta}\left(r=r_{0}\right) & =\frac{1}{r_{0}} \sum_{n, m}^{\prime}\left(\psi_{n m}^{\prime}+\frac{(n+3)}{2 \eta^{\prime}(n+1)(2 n+3)} r_{0}^{2} P_{n m}^{\prime}\right) \frac{\partial Y_{n m}}{\partial \theta} e^{i \omega t} \\
& =\frac{1}{r_{0}} \sum_{n, m}^{\prime}\left(\psi_{n m}-\frac{(n-2)}{2 \eta n(2 n-1)} r_{0}^{2} P_{n m}\right) \frac{\partial Y_{n m}}{\partial \theta} e^{i \omega t}+v_{\theta}^{\infty}\left(r=r_{0}\right) .
\end{aligned}
$$

The continuity condition in the $\boldsymbol{e}_{\varphi}$-direction results in the equivalent condition as $\mathrm{Eq}$. (4.3).

An additional set of boundary conditions follows from the balance of force on the membrane presented by Eq. (2.21). By noticing that the Laplace-Beltrami operator is now the usual Laplacian operator on the sphere

$$
\nabla_{L B}^{2}=\frac{\nabla_{1}^{2}}{r_{0}^{2}}
$$

the force balance equations in the $\boldsymbol{e}_{r}$ and $\boldsymbol{e}_{\theta}$-directions up to first order in terms of $\ell$ are given by

$$
\begin{aligned}
& \left(P-P^{\prime}\right)-\left(2 \eta \frac{\partial v_{r}}{\partial r}-2 \eta^{\prime} \frac{\partial v_{r}^{\prime}}{\partial r}\right)+\frac{2}{r_{0}} \delta \Sigma\left(\rho_{s}\right) \\
& -\frac{1}{r_{0}^{4}}\left[\Sigma\left(\rho_{s 0}\right) r_{0}^{2}-\kappa\left(\nabla_{\perp}^{2}+2 c_{0} r_{0}-\frac{1}{2} c_{0}^{2} r_{0}^{2}\right)\right]\left(2+\nabla_{\perp}^{2}\right) \ell(\theta, \varphi, t)=0
\end{aligned}
$$

and

$$
-\eta\left(\frac{1}{r_{0}} \frac{\partial v_{r}}{\partial \theta}+\frac{\partial v_{\theta}}{\partial r}-\frac{v_{\theta}}{r_{0}}\right)+\eta^{\prime}\left(\frac{1}{r_{0}} \frac{\partial v_{r}^{\prime}}{\partial \theta}+\frac{\partial v_{\theta}^{\prime}}{\partial r}-\frac{v_{\theta}^{\prime}}{r_{0}}\right)-\frac{1}{r_{0}} \frac{\partial}{\partial \theta} \delta \Sigma\left(\rho_{s}\right)=0,
$$

at $r=r_{0}$, respectively. In the above, we used the capillarity condition Eq. (3.20). Notice also that $n_{r} \approx 1$, while $n_{\theta} \approx-\left(1 / r_{0}\right)(\partial \ell / \partial \theta)$, which is proportional to $\ell$ to the lowest order. In order to eliminate $\delta \Sigma\left(\rho_{s}\right)$ from the above equations, we obtain the following relation from Eqs. (2.17), (2.31) and (4.1):

$$
\frac{\partial}{\partial t} \delta \Sigma\left(\rho_{s}\right)=B \operatorname{div}_{\perp} v^{\prime}+\frac{2 B}{r_{0}} \frac{\partial \ell}{\partial t}
$$




$$
\begin{aligned}
= & -\frac{B}{r_{0}^{2}} \sum_{n, m}^{\prime} n(n+1)\left(\psi_{n m}^{\prime}+\frac{n+3}{2 \eta^{\prime}(n+1)(2 n+3)} P_{n m}^{\prime} r_{0}^{2}\right) Y_{n m}(\theta, \varphi) e^{i \omega t} \\
& +i \omega \frac{2 B}{r_{0}} \sum_{n, m}^{\prime} \ell_{n m} Y_{n m}(\theta, \varphi) e^{i \omega t}
\end{aligned}
$$

at $r=r_{0}$. Substitution of Eq. (4.7) into the time derivative of Eqs. (4.5) and (4.6) yields

$$
\begin{aligned}
i \omega & {\left[P_{m m}^{\prime}-\left(2 \eta^{\prime} n(n-1) \frac{\psi_{n m}^{\prime}}{r_{0}^{2}}-2 \eta n(n-1) \Gamma+\frac{n(n+1)}{2 n+3} P_{n m}^{\prime}\right)\right] } \\
& -i \omega\left[P_{n m}-\left(2 \eta(n+1)(n+2) \frac{\psi_{n m}}{r_{0}^{2}}-\frac{n(n+1)}{2 n-1} P_{n m}\right)\right] \\
& -i \omega \frac{1}{r_{0}^{4}}(n-1)(n+2)\left\{\Sigma\left(\rho_{s 0}\right) r_{0}^{2}+\kappa\left[(n+1)-2 c_{0} r_{0}+\frac{1}{2} c_{0}^{2} r_{0}^{2}\right]\right\} \ell_{n m} \\
& +\frac{2 B}{r_{0}^{3}} n(n+1)\left(\psi_{n m}^{\prime}+\frac{n+3}{2 \eta^{\prime}(n+1)(2 n+3)} P_{n m}^{\prime} r_{0}^{2}\right)-i \omega \frac{4 B}{r_{0}^{2}} \ell_{n m}=0,
\end{aligned}
$$

and

$$
\begin{aligned}
& i \omega\left(2 \eta^{\prime}(n-1) \frac{\psi_{n m}^{\prime}}{r_{0}^{2}}-2 \eta(n-1) \Gamma+\frac{n(n+2)}{(n+1)(2 n+3)} P_{n m}^{\prime}\right) \\
& \quad+i \omega\left(2 \eta(n+2) \frac{\psi_{n m}}{r_{0}^{2}}-\frac{(n-1)(n+1)}{n(2 n-1)} P_{n m}\right) \\
& \quad+\frac{B}{r_{0}^{3}} n(n+1)\left(\psi_{n m}^{\prime}+\frac{n+3}{2 \eta^{\prime}(n+1)(2 n+3)} P_{n m}^{\prime} r_{0}^{2}\right)-i \omega \frac{2 B}{r_{0}^{2}} \ell_{n m}=0
\end{aligned}
$$

respectively, for $n \geq 1$.

As far as we consider the shearing motion given by Eq. (3.25), it is sufficient to take only $(n, m)=(2,0)$ mode in the above conditions. Introducing the abbreviation

$$
S \equiv \Sigma\left(\rho_{s 0}\right)+\frac{\kappa}{r_{0}^{2}}\left(6-2 c_{0} r_{0}+\frac{1}{2} c_{0}^{2} r_{0}^{2}\right)
$$

and omitting the suffixes 20 of all the functions, Eqs. (4.2), (4.3), (4.8), (4.9) reduce to the following equations to be solved (we have also eliminated $\ell_{20}$ ):

$$
\begin{aligned}
& \frac{1}{7 \eta^{\prime}} P^{\prime}+\frac{2}{r_{0}^{2}} \psi^{\prime}=\frac{1}{2 \eta} P-\frac{3}{r_{0}^{2}} \psi+2 \Gamma, \\
& \frac{5}{14 \eta^{\prime}} P^{\prime}+\frac{3}{r_{0}^{2}} \psi^{\prime}=\frac{3}{r_{0}^{2}} \psi+3 \Gamma, \\
& \frac{1}{7 \eta^{\prime} r_{0}^{2}}\left(4 r_{0} S-6 r_{0} B-i \omega \eta^{\prime} r_{0}^{2}\right) P^{\prime}+\frac{4}{r_{0}^{2}}\left(\frac{2 S}{r_{0}}-\frac{B}{r_{0}}+i \omega \eta^{\prime}\right) \psi^{\prime} \\
& =i \omega \eta\left(-\frac{3}{\eta} P+\frac{24}{r_{0}^{2}} \psi+4 \Gamma\right),
\end{aligned}
$$




$$
\begin{aligned}
& \frac{1}{7 \eta^{\prime} r_{0}^{2}}\left(9 r_{0} B+8 i \omega \eta^{\prime} r_{0}^{2}\right) P^{\prime}+\frac{6}{r_{0}^{2}}\left(\frac{B}{r_{0}}+i \omega \eta^{\prime}\right) \psi^{\prime} \\
& \quad=i \omega \eta\left(\frac{3}{2 \eta} P-\frac{24}{r_{0}^{2}} \psi+6 \Gamma\right) .
\end{aligned}
$$

The set of these four linear homogeneous equations in the five unknowns $P_{20}^{\prime}, \psi_{20}^{\prime}$, $P_{20}, \psi_{20}$ and $\Gamma$ provide sufficient information to calculate $P_{20} / \Gamma$ from which we can calculate the effective viscosity according to Eq. (3.32). After some calculation, the final expression is obtained as

$$
\begin{aligned}
& \eta^{*} / \eta=1 \\
& \quad+5 \frac{24 B S+[(23 E-16) B+4(5 E+2) S] f+(E-1)(19 E+16) f^{2}}{48 B S+[2(23 E+32) B+40(E+1) S] f+(2 E+3)(19 E+16) f^{2}} \phi,
\end{aligned}
$$

where $E=\eta^{\prime} / \eta, f=i \omega r_{0} \eta$.

The equation which determines the overdamped mode of the membrane deformation can be obtained by putting the denominator of Eq. (4.15) equal to zero. When neither $B$ nor $S$ is zero $(B \neq 0$ and $S \neq 0$ ), it exhibits, in general, two characteristic times. On the other hand, in the case of $B=S=0$, we find a stationary $\eta^{*}$ given by

$$
\eta^{*} / \eta=1+\frac{5(E-1)}{2 E+3} \phi
$$

which was first derived by Taylor [36]. In such a case there is no force due to the membrane and the fluid stress tensors balance at the interface. The previous argument leading to Eq. (3.12) corresponds to this case and we see that the viscosity change is proportional to $\eta-\eta^{\prime}$ as it should be [55].

When either one of $B$ or $S$ is zero or tends to infinity, only a single characteristic time shows up. This case can be represented in general by

$$
\eta^{*}=\eta_{\infty}+\frac{G \tau}{1+i \omega \tau}
$$

which coincides with the outcome of the "Maxwell model" in the phenomenological rheology. In the above, $\tau$ is the relaxation time, $G$ the relaxation strength and $\eta_{\infty}$ is the constant viscosity when $\omega \rightarrow \infty . \tau$ represents the time scale dividing the short time Hookian regime and the long time Newtonian regime. As we show in Appendix $\mathrm{D}, \tau$ corresponds to the longest relaxation time of the membrane deformation for each limiting case. We shall collect below the results of $\eta_{\infty}, \tau, G$ and $\eta_{0}^{*}=\lim _{\omega \rightarrow 0} \eta^{*}$ for several cases depending on the relation between $B$ and $S$.

(i) $B \gg S>0$

This limit corresponds to the case where the membrane is incompressible. Taking the limit of $B / S \rightarrow \infty$ in Eq. (4.15), we find

$$
\eta_{\infty} / \eta=1+\frac{5(23 E-16)}{2(23 E+32)} \phi
$$




$$
\begin{aligned}
\tau & =\frac{\eta r_{0}}{S} \frac{23 E+32}{24}, \\
G & =\frac{S}{r_{0}} \frac{2880}{(23 E+32)^{2}} \phi,
\end{aligned}
$$

and

$$
\eta_{0}^{*} / \eta=1+\frac{5}{2} \phi
$$

Eq. (4.21) does not depend on the fluid viscosity and coincides with that of dilute suspensions of rigid particles [39].

(ii) $S \gg B>0$

In the limit of $S / B \rightarrow \infty$, we have

$$
\begin{aligned}
& \eta_{\infty} / \eta=1+\frac{5 E+2}{2(E+1)} \phi, \\
& \tau=\frac{\eta r_{0}}{B} \frac{5(E+1)}{6}, \\
& G=\frac{B}{r_{0}} \frac{9}{5(E+1)^{2}} \phi,
\end{aligned}
$$

and $\eta_{0}^{*}$ is identical to Eq. (4.21).

(iii) $S>B=0$

In this limit the membrane is fully compressible. By putting $B=0$, we have

$$
\begin{aligned}
& \eta_{\infty} / \eta=1+\frac{5(E-1)}{2 E+3} \phi \\
& \tau=\frac{\eta r_{0}}{S} \frac{(2 E+3)(19 E+16)}{40(E+1)} \\
& G=\frac{S}{r_{0}} \frac{20}{(2 E+3)^{2}} \phi
\end{aligned}
$$

and

$$
\eta_{0}^{*} / \eta=1+\frac{5 E+2}{2(E+1)} \phi
$$

Eq. (4.28) was first derived by Taylor for the dispersions of almost undeformable liquid droplets with a large surface tension [35]. The case of $E=1 \mathrm{in} \mathrm{Eq.} \mathrm{(4.28)}$ was also discussed by Schwartz and Edwards [60,61].

(iv) $B>S=0$

In this case we have

$$
\begin{aligned}
& \eta_{\infty} / \eta=1+\frac{5(E-1)}{2 E+3} \phi \\
& \tau=\frac{\eta r_{0}}{B} \frac{(2 E+3)(19 E+16)}{2(23 E+32)},
\end{aligned}
$$




$$
G=\frac{B}{r_{0}} \frac{5}{(2 E+3)^{2}} \phi,
$$

and

$$
\eta_{0}^{*} / \eta=1+\frac{5(23 E-16)}{2(23 E+32)} \phi .
$$

In Appendix D, we have listed the sequence of the relaxation time of the membrane deformation for general $n$ in accordance with the cases considered here. Notice that all the relaxation times given above are recovered by putting $n=2$ in the corresponding expressions.

Another limit can be taken in the small frequency limit by neglecting the $f^{2}$ terms in Eq. (4.15). In this case, the effective viscosity can be also expressed in terms of the Maxwell model, Eq. (4.17), with

$$
\begin{aligned}
& \eta_{\infty} / \eta=1+\frac{5[(23 E-16) B+4(5 E+2) S]}{2[(23 E+32) B+20(E+1) S]} \phi, \\
& \tau=\frac{\eta r_{0}}{B S} \frac{(23 E+32) B+20(E+1) S}{24}, \\
& G=\frac{B S}{r_{0}} \frac{720(4 B+S)}{[(23 E+32) B+20(E+1) S]^{2}} \phi,
\end{aligned}
$$

and $\eta_{0}^{*}$ is identical to Eq. (4.21).

In all these cases, both $\eta_{\infty}$ and $\eta_{0}^{*}$ approach to Einstein's result by taking the limit of $\eta^{\prime} \rightarrow \infty$. It should be emphasized that as long as the membrane is characterized by the non-zero $B$ and $S$, the steady state effective viscosity coincides with that of a suspension of solid particles [52,62]. Moreover, neither $B$ nor $S$ appears in the steady state viscosity, while the relaxation time and the relaxation strength depend explicitly on $B$ and $S$. Hence an appropriate dynamical experiment should be performed in order to grasp the elastic properties of the membrane. Although these statements might appear obvious in the early calculations by Oldroyd [31,32], it should be stressed that he did not take into account the effect of the bending rigidity $\kappa$. We also comment that the recent work by Onuki agrees with our calculation [26].

\section{Nondilute case}

In the previous section, we have analyzed the dilute case which is valid only when the droplet concentration is sufficiently low and the effect of droplets on the macroscopic properties scales linearly with the volume fraction. As the volume fraction increases, such a dilute picture breaks down and the presence of the droplets affects the flow field around the other droplets.

As far as the stationary effective viscosity is concerned, studies of nondilute suspensions of rigid particles are reviewed in the book by Happel and Brenner [58], and 


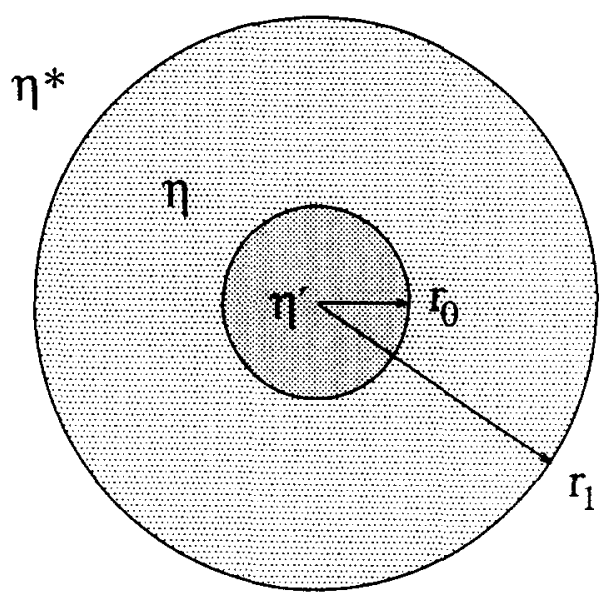

Fig. 1. Schematic representation of the cell model.

notable among them is what is called the "cell models" used by Simha [63] and Happel [64]. In this model the hydrodynamic interactions between particles are taken into account to some extent. The result obtained by using Simha's model reduces to that given by Einstein's theory for a very dilute system [65]. However, Happel's prediction is larger than both Einstein's and Simha's results even for small concentrations. This trend is reversed at large concentrations when the theoretical viscosity is compared with the experimental data. In the case of nondilute emulsions, Choi and Schowalter proceeded the analysis by means of Simha's cell model [41]. In this section, we shall follow their scheme to calculate the effective viscosity of a nondilute dispersion.

In the cell model, we have extra boundary conditions at $r=r_{1}>r_{0}$, where the velocity is equal to the velocity of a homogeneous system (see Fig. 1). $r_{1}$ can be regarded as the hydrodynamic interaction radius. The results of the dilute case are recovered by taking the limit of $r_{1} \rightarrow \infty$. The velocity field inside of the droplet, $r \leq r_{0}$, is given by Eq. (3.22) and the fluid at $r \geq r_{1}$ is described by the flow Eq. (3.26). In-between $\left(r_{0} \leq r \leq r_{1}\right)$ the velocity field is expressed as a superposition of Eqs. (3.22) and (3.23):

$$
\begin{aligned}
v(\boldsymbol{r}, t)= & \sum_{n, m}^{\prime}\left[\left(\psi_{n m}^{\prime \prime} \operatorname{grad}+\frac{(n+3)}{2 \eta(n+1)(2 n+3)} P_{n m}^{\prime \prime} r^{2} \mathrm{grad}\right.\right. \\
& \left.-\frac{n}{\eta(n+1)(2 n+3)} P_{n m}^{\prime \prime} \boldsymbol{r}\right)\left(\frac{r}{r_{0}}\right)^{n} \\
& +\left(\psi_{n m} \operatorname{grad}-\frac{(n-2)}{2 \eta n(2 n-1)} P_{n m} r^{2} \operatorname{grad}\right. \\
& \left.\left.+\frac{(n+1)}{\eta n(2 n-1)} P_{n m} r\right)\left(\frac{r_{0}}{r}\right)^{(n+1)}\right] Y_{n m}(\theta, \varphi) e^{i \omega t}
\end{aligned}
$$

The first set of boundary conditions comes from the continuity of the velocity field at 
$r=r_{0}$. In the $e_{r}$-direction, this is written as

$$
\begin{aligned}
v_{r}\left(r=r_{0}\right)= & \frac{1}{r_{0}} \sum_{n, m}^{\prime}\left(n \psi_{n m}^{\prime}+\frac{n}{2 \eta^{\prime}(2 n+3)} r_{0}^{2} P_{n m}^{\prime}\right) Y_{n m}(\theta, \varphi) e^{i \omega t} \\
= & -\frac{1}{r_{0}} \sum_{n, m}^{\prime}\left(-n \psi_{n m}^{\prime \prime}-\frac{n}{2 \eta(2 n+3)} r_{0}^{2} P_{n m}^{\prime \prime}\right. \\
& \left.+(n+1) \psi_{n m}-\frac{(n+1)}{2 \eta(2 n-1)} r_{0}^{2} P_{n m}\right) Y_{n m}(\theta, \varphi) e^{i \omega t} \\
= & \sum_{n, m}^{\prime} i \omega \ell_{n m} Y_{n m}(\theta, \varphi) e^{i \omega t},
\end{aligned}
$$

where the last expression has been obtained by taking the time derivative of Eq. (2.33). Likewise, the continuity of the velocity in the $\boldsymbol{e}_{\theta}$-direction yields

$$
\begin{aligned}
v_{\theta}\left(r=r_{0}\right)= & \frac{1}{r_{0}} \sum_{n, m}^{\prime}\left(\psi_{n m}^{\prime}+\frac{(n+3)}{2 \eta^{\prime}(n+1)(2 n+3)} r_{0}^{2} P_{n m}^{\prime}\right) \frac{\partial Y_{n m}}{\partial \theta} e^{i \omega t} \\
= & \frac{1}{r_{0}} \sum_{n, m}^{1}\left(\psi_{n m}^{\prime \prime}+\frac{(n+3)}{2 \eta(n+1)(2 n+3)} r_{0}^{2} P_{n m}^{\prime \prime}\right. \\
& \left.+\psi_{n m}-\frac{(n-2)}{2 \eta n(2 n-1)} r_{0}^{2} P_{n m}\right) \frac{\partial Y_{n m}}{\partial \theta} e^{i \omega t} .
\end{aligned}
$$

The other set of boundary conditions follows from the balance of forces on the membrane given by Eq. (2.21). Similar to the previous section, we have

$$
\begin{aligned}
i \omega & {\left[\left(P_{n m}^{\prime}-P_{n m}^{\prime \prime}\right)-2 n(n-1)\left(\frac{\eta^{\prime} \psi_{n m}^{\prime}}{r_{0}^{2}}-\frac{\eta \psi_{n m}^{\prime \prime}}{r_{0}^{2}}\right)-\frac{n(n+1)}{2 n+3}\left(P_{n m}^{\prime}-P_{n m}^{\prime \prime}\right)\right] } \\
& -i \omega\left[P_{n m}-\left(2 \eta(n+1)(n+2) \frac{\psi_{n m}}{r_{0}^{2}}-\frac{n(n+1)}{2 n-1} P_{n m}\right)\right] \\
& -i \omega \frac{1}{r_{0}^{4}}(n-1)(n+2)\left\{\Sigma\left(\rho_{s 0}\right) r_{0}^{2}+\kappa\left[(n+1)-2 c_{0} r_{0}+\frac{1}{2} c_{0}^{2} r_{0}^{2}\right]\right\} \ell_{n m} \\
& +\frac{2 B}{r_{0}^{3}} n(n+1)\left(\psi_{n m}^{\prime}+\frac{n+3}{2 \eta^{\prime}(n+1)(2 n+3)} P_{n m}^{\prime} r_{0}^{2}\right)-i \omega \frac{4 B}{r_{0}^{2}} \ell_{n m}=0,
\end{aligned}
$$

and

$$
\begin{aligned}
i \omega & {\left[2(n-1)\left(\frac{\eta^{\prime} \psi_{n m}^{\prime}}{r_{0}^{2}}-\frac{\eta \psi_{n m}^{\prime \prime}}{r_{0}^{2}}\right)+\frac{n(n+2)}{(n+1)(2 n+3)}\left(P_{n m}^{\prime}-P_{n m}^{\prime \prime}\right)\right] } \\
& +i \omega\left(2 \eta(n+2) \frac{\psi_{n m}}{r_{0}^{2}}-\frac{(n-1)(n+1)}{n(2 n-1)} P_{n m}\right) \\
& +\frac{B}{r_{0}^{3}} n(n+1)\left(\psi_{n m}^{\prime}+\frac{n+3}{2 \eta^{\prime}(n+1)(2 n+3)} P_{n m}^{\prime} r_{0}^{2}\right)-i \omega \frac{2 B}{r_{0}^{2}} \ell_{n m}=0
\end{aligned}
$$

for $n \geq 1$. 
In Simha's cell model, the velocity is required to be continuous at $r=r_{1}$. Hence the conditions are for $\boldsymbol{e}_{r}$ and $\boldsymbol{e}_{\theta}$-direction as

$$
\begin{aligned}
v_{r}\left(r=r_{1}\right)= & {\left[\frac{1}{r_{0}} \sum_{n, m}^{\prime}\left(\frac{r_{1}}{r_{0}}\right)^{n-1}\left(n \psi_{n m}^{\prime \prime}+\frac{n}{2 \eta(2 n+3)} r_{1}^{2} P_{n m}^{\prime \prime}\right)\right.} \\
& \left.-\frac{1}{r_{1}} \sum_{n, m}^{\prime}\left(\frac{r_{0}}{r_{1}}\right)^{n+1}\left((n+1) \psi_{n m}-\frac{(n+1)}{2 \eta(2 n-1)} r_{1}^{2} P_{n m}\right)\right] Y_{n m}(\theta, \varphi) e^{i \omega t} \\
= & \sum_{n, m}^{\prime} n r_{1}^{n-1} \Gamma Y_{n m}(\theta, \varphi) e^{i \omega t}
\end{aligned}
$$

and

$$
\begin{aligned}
v_{\theta}\left(r=r_{1}\right)= & {\left[\frac{1}{r_{0}} \sum_{n, m}^{\prime}\left(\frac{r_{1}}{r_{0}}\right)^{n-1}\left(\psi_{n m}^{\prime \prime}+\frac{(n+3)}{2 \eta(n+1)(2 n+3)} r_{0}^{2} P_{n m}^{\prime \prime}\right)\right.} \\
& \left.+\frac{1}{r_{1}} \sum_{n, m}^{\prime}\left(\frac{r_{0}}{r_{1}}\right)^{n+1}\left(\psi_{n m}-\frac{(n-2)}{2 \eta n(2 n-1)} r_{0}^{2} P_{n m}\right)\right] \frac{\partial Y_{n m}}{\partial \theta} e^{i \omega t} \\
= & \sum_{n, m}^{\prime} r_{1}^{n-1} \Gamma \frac{\partial Y_{n m}}{\partial \theta} e^{i \omega t},
\end{aligned}
$$

respectively.

As before, it is sufficient to take only $(n, m)=(2,0)$ mode in the above conditions. Using the abbreviation of Eq. (4.10) and omitting the suffixes 20 of all the functions, Eqs. (5.2), (5.3), (5.4), (5.5), (5.6) and (5.7) are reduced to the following equations to be solved:

$$
\begin{aligned}
& \frac{1}{7 \eta^{\prime}} P^{\prime}+\frac{2}{r_{0}^{2}} \psi^{\prime}=\frac{1}{7 \eta} P^{\prime \prime}+\frac{2}{r_{0}^{2}} \psi^{\prime \prime}+\frac{1}{2 \eta} P-\frac{3}{r_{0}^{2}} \psi, \\
& \frac{5}{14 \eta^{\prime}} P^{\prime}+\frac{3}{r_{0}^{2}} \psi^{\prime}=\frac{5}{14 \eta} P^{\prime \prime}+\frac{3}{r_{0}^{2}} \psi^{\prime \prime}+\frac{3}{r_{0}^{2}} \psi, \\
& \frac{1}{7 \eta^{\prime} r_{0}^{2}}\left(4 r_{0} S-6 r_{0} B-i \omega \eta^{\prime} r_{0}^{2}\right) P^{\prime}+\frac{4}{r_{0}^{2}}\left(\frac{2 S}{r_{0}}-\frac{B}{r_{0}}+i \omega \eta^{\prime}\right) \psi^{\prime} \\
& =i \omega \eta\left(-\frac{1}{7 \eta} P^{\prime \prime}+\frac{4}{r_{0}^{2}} \psi^{\prime \prime}\right)+i \omega \eta\left(-\frac{3}{\eta} P+\frac{24}{r_{0}^{2}} \psi\right), \\
& \frac{1}{7 \eta^{\prime} r_{0}^{2}}\left(9 r_{0} B+8 i \omega \eta^{\prime} r_{0}^{2}\right) P^{\prime}+\frac{6}{r_{0}^{2}}\left(\frac{B}{r_{0}}+i \omega \eta^{\prime}\right) \psi^{\prime} \\
& =i \omega \eta\left(\frac{8}{7 \eta} P^{\prime \prime}+\frac{6}{r_{0}^{2}} \psi^{\prime \prime}\right)+i \omega \eta\left(\frac{3}{2 \eta} P-\frac{24}{r_{0}^{2}} \psi\right),
\end{aligned}
$$




$$
\begin{aligned}
& \frac{1}{7 \eta}\left(\frac{r_{1}}{r_{0}}\right)^{2} P^{\prime \prime}+\frac{2}{r_{0}^{2}} \psi^{\prime \prime}+\frac{1}{2 \eta}\left(\frac{r_{0}}{r_{1}}\right)^{3} P-\frac{3}{r_{1}^{2}}\left(\frac{r_{0}}{r_{1}}\right)^{3} \psi=2 \Gamma, \\
& \frac{5}{14 \eta}\left(\frac{r_{1}}{r_{0}}\right)^{2} P^{\prime \prime}+\frac{3}{r_{0}^{2}} \psi^{\prime \prime}+\frac{3}{r_{1}^{2}}\left(\frac{r_{0}}{r_{1}}\right)^{3} \psi=3 \Gamma .
\end{aligned}
$$

The set of these six linear homogeneous equations for the seven unknowns $P_{20}^{\prime}, \psi_{20}^{\prime}$, $P_{20}, \psi_{20}, P_{20}^{\prime \prime}, \psi_{20}^{\prime \prime}$ and $\Gamma$ are sufficient to calculate $P_{20} / \Gamma$ from which we calculate the effective viscosity Eq. (3.32) as before. After a tedious calculation, the effective viscosity is expressed by introducing the notations $f=i \omega r_{0} \eta, E=\eta^{\prime} / \eta$ and $y=r_{0} / r_{1}$ as

$$
\eta^{*} / \eta=1+10 \frac{C_{4}+C_{5} f+C_{6} f^{2}}{C_{1}+C_{2} f+C_{3} f^{2}} \phi,
$$

where

$$
\begin{aligned}
C_{1}= & 24 B S\left(4-25 y^{3}+42 y^{5}-25 y^{7}+4 y^{10}\right), \\
C_{2}= & 4[(23 E+32) B+20(E+1) S] \\
& -25[(23 E-16) B+4(5 E+2) S] y^{3} \\
& +42[(23 E-8) B+20 E S] y^{5} \\
& -25[(23 E+4) B+4(5 E-2) S] y^{7} \\
& +4(E-1)(23 B+20 S) y^{10}, \\
C_{3}= & 2(2 E+3)(19 E+6)-25(E-1)(19 E+16) y^{3} \\
& +49(E-1)(19 E+16) y^{5}-25(E-1)(19 E+18) y^{7} \\
& +76(E-1)^{2} y^{10}, \\
C_{4}= & 24 B S\left(1-y^{7}\right), \\
C_{5}= & (23 E-16) B+4(5 E+2) S-(E-1)(23 B+20 S) y^{7}, \\
C_{6}= & (E-1)(19 E+16)-19(E-1)^{2} y^{7} .
\end{aligned}
$$

Notice that $y=r_{0} / r_{1}$ introduces the effect of interaction among droplets. In the limit of $y \rightarrow 0$, the above results reduce to Eq. (4.15) of the dilute case. Since the interactions between the droplets are effectively accounted for by the introduction of the radius $r_{1}$, $y$ can be an explicit function of the volume fraction $\phi$, such as $[42,63]$

$$
y^{3}=g \phi
$$

where $g$ is a constant number. The Oldroyd's result is recovered when $g=(2 / 5)^{2}$ [31]. Choi and Schowalter, on the other hand, chose $g=1$ [41] and Thomas proposed $g=0.656$ for $\phi \leq 0.1[66]$.

As regards the characteristic relaxation times, they satisfy the equations obtained by putting the numerator and denominator of Eq. (5.14) equal to zero. When neither $B$ 
nor $S$ is zero ( $B \neq 0$ and $S \neq 0$ ), each equation determines two characteristic times. In this case, we can write the effective viscosity in the form

$$
\eta^{*}=\eta_{0}^{*} \frac{1+i \omega \tau_{2}-\omega^{2} \nu_{2}}{1+i \omega \tau_{1}-\omega^{2} \nu_{1}},
$$

where

$$
\begin{aligned}
\eta_{0}^{*} / \eta & =1+\frac{10\left(1-y^{7}\right)}{4-25 y^{3}+42 y^{5}-25 y^{7}+4 y^{10}} \phi \\
& \approx 1+\frac{5}{2} \phi\left(1+\frac{25}{4} g \phi\right) \\
\tau_{1} \approx & \frac{\eta r_{0}}{B S}\left(\frac{(23 E+32) B+20(E+1) S}{24}+\frac{25(4 B+S)}{8} g \phi\right), \\
\tau_{2} \approx & \frac{\eta r_{0}}{B S}\left(\frac{(23 E+32) B+20(E+1) S}{24}+\frac{5(4 B+S)}{8}(5 g-2) \phi\right), \\
\nu_{1} \approx & \frac{\left(\eta r_{0}\right)^{2}}{B S} \frac{(2 E+3)(19 E+16)}{48}\left(1+\frac{125}{4(2 E+3)} g \phi\right) \\
\nu_{2} \approx & \frac{\left(\eta r_{0}\right)^{2}}{B S} \frac{(2 E+3)(19 E+16)}{48}\left(1+\frac{25}{4(2 E+3)}(5 g-2) \phi\right) .
\end{aligned}
$$

Eq. (5.18) coincides with the steady state viscosity of a suspension of rigid spheres derived by Simha [63]. In the absence of the bending rigidity, $\kappa=0$, this result can be included as a special case of that given by Oosterbroek and Mellema, where more complex mechanical properties such as the viscosity and shear elasticity of the film were incorporated [42]. On the other hand, in the case of $B=S=0$ we find a stationary $\eta^{*}$ given by

$$
\begin{aligned}
\eta^{*} / \eta & =1+10 \frac{C_{6}}{C_{3}} \phi \\
& \approx 1+\frac{5(E-1)}{2 E+3} \phi\left(1+\frac{25(E-1)}{2(2 E+3)} g \phi\right) .
\end{aligned}
$$

When either one of $B$ or $S$ is zero or tends to infinite, the effective viscosity can be written formally in the following form

$$
\eta^{*}=\eta_{0}^{*} \frac{1+i \omega \tau_{2}}{1+i \omega \tau_{1}}
$$

where $\tau_{1}, \tau_{2}$ are the characteristic times. We give below the explicit expressions of $\eta_{0}^{*}$, $\tau_{1}$ and $\tau_{2}$ according to the relation between $B$ and $S$.

(i) $B \gg S>0$

$\eta_{0}^{*}$ is identical to Eq. (5.18),

$$
\tau_{1} \approx \frac{\eta r_{0}}{S}\left(\frac{23 E+32}{24}+\frac{25}{2} g \phi\right),
$$




$$
\tau_{2} \approx \frac{\eta r_{0}}{S}\left(\frac{23 E+32}{24}+\frac{5}{2}(5 g-2) \phi\right) \text {. }
$$

(ii) $S \gg B>0$

$\eta_{0}^{*}$ is identical to Eq. (5.18),

$$
\begin{aligned}
& \tau_{1} \approx \frac{\eta r_{0}}{B}\left(\frac{5(E+1)}{6}+\frac{25}{8} g \phi\right), \\
& \tau_{2} \approx \frac{\eta r_{0}}{B}\left(\frac{5(E+1)}{6}+\frac{5}{8}(5 g-2) \phi\right) .
\end{aligned}
$$

(iii) $S>B=0$

$$
\begin{aligned}
& \eta_{0}^{*} / \eta=1 \\
& +\frac{2(5 E+2)-10(E-1) y^{7}}{4(E+1)-5(5 E+2) y^{3}+42 E y^{5}-5(5 E-2) y^{7}+4(E-1) y^{10}} \phi \\
& \quad \approx 1+\frac{5 E+2}{2(E+1)} \phi\left(1+\frac{5(5 E+2)}{4(E+1)} g \phi\right) \\
& \tau_{1} \approx \frac{\eta r_{0}}{S} \frac{(2 E+3)(19 E+16)}{40(E+1)}\left(1+\frac{5}{4} \frac{19 E+16}{(E+1)(2 E+3)} g \phi\right) \\
& \tau_{2} \approx \frac{\eta r_{0}}{S} \frac{(2 E+3)(19 E+16)}{40(E+1)}\left(1+\frac{1}{4} \frac{19 E+16}{(E+1)(2 E+3)}(5 g-2) \phi\right)
\end{aligned}
$$

(iv) $B>S=0$

$$
\begin{aligned}
\eta_{0}^{*} / \eta= & 1+\left[10(23 E-16)-230(E-1) y^{7}\right] \\
& /\left[4(23 E+32)-25(23 E-16) y^{3}+42(23 E-8) y^{5}\right. \\
& \left.-25(23 E+4) y^{7}+92(E-1) y^{10}\right] \phi \\
\approx & 1+\frac{5(23 E-16)}{2(23 E+32)} \phi\left(1+\frac{25(23 E-16)}{4(23 E+32)} g \phi\right), \\
\tau_{1} \approx & \frac{\eta r_{0}}{B} \frac{(2 E+3)(19 E+16)}{2(23 E+32)}\left(1+\frac{25}{4} \frac{19 E+16}{(23 E+32)(2 E+3)} g \phi\right), \\
\tau_{2} \approx & \frac{\eta r_{0}}{B} \frac{(2 E+3)(19 E+16)}{2(23 E+32)}\left(1+\frac{5}{4} \frac{19 E+16}{(23 E+32)(2 E+3)}(5 g-2) \phi\right) .
\end{aligned}
$$

Eq. (5.29) was derived by several authors by means of various methods $[41,67,68]$. 
Before closing this section, we point out that the relaxation time $\tau$ and the relaxation strength $G$ in the Maxwell model Eq. (4.17) can be related to the quantities discussed in this section. In fact, $\tau$ corresponds $\tau_{1}$ while $G$ can be written as

$$
G=\eta_{0}^{*}\left(\tau_{1}-\tau_{2}\right) / \tau_{1}^{2},
$$

which characterizes the elastic modulus for $t \ll \tau$ [69]. Thus $\tau_{1}-\tau_{2}$ is proportional to the elastic part of the stress tensor [40].

\section{Discussion}

In this paper, we have examined the effective dynamical viscosity of dispersions of fluid droplets coated by amphiphilic molecules. The shape of the fluid membrane is considered to be mainly governed by the compression modulus $B$ and the bending rigidity $\kappa$. We obtained its general expression in terms of $B$ and $S$ (Eq. (4.10)) both for dilute and nondilute cases. Several limiting cases have been also derived. The time scale of the viscoelastic behavior is characterized by the longest relaxation time of the membrane deformation when either one of $B$ or $S$ is zero or infinity. According to the analysis of the dilute limit, it is found that when both $B$ and $S$ are non-zero, the stationary effective viscosity $\eta_{0}^{*}$ reduces to Einstein's result. In case either one of $B$ or $S$ is zero, $\eta_{0}^{*}$ depends on the viscosity of the inside fluid $\eta^{\prime}$ while Taylor's result can be reproduced when $B=0$ and $S \neq 0$. A cell model for the nondilute case yields Simha's result for $\eta_{0}^{*}$ when neither $B$ nor $S$ is zero.

In order to estimate the value of the bending rigidity $\kappa$ from the observed characteristic time, we have to eliminate $\Sigma\left(\rho_{s 0}\right)$ from $S$ (see Eq. (4.10)). (For the present discussion, we ignore the spontaneous curvature $c_{0}$.) This can be done by an independent observation of $\Sigma\left(\rho_{s 0}\right)$ according to the "capillarity condition" Eq. (3.20) [9]. In practice, however, it is difficult to perform such a measurement in a sufficient accuracy and many people assumed that $\Sigma\left(\rho_{s 0}\right) \simeq 0$. In such a case, it is possible to estimate $\kappa$ from the measured relaxation time of the membrane deformation [5-12]. Such a simplification can be justified provided $\Sigma\left(\rho_{s 0}\right) \ll \kappa / r_{0}^{2}$ is satisfied. The quantitative estimation of this condition should be done separately for bilayer vesicles and for microemulsion droplets since they differ by several decades in length scale.

For a lipid vesicle of size $r_{0} \simeq 10^{-5}[\mathrm{~m}]$, various people observed that the bending rigidity is typically $\kappa \simeq 1 \times 10^{-19}[\mathrm{~J}][5-12]$ and hence we have $\kappa / r_{0}^{2} \simeq 1 \times 10^{-9}$ $[\mathrm{N} / \mathrm{m}]$. As for the surface tension, some people claim that it is less than $10^{-5}[\mathrm{~N} / \mathrm{m}]$ [70] or $10^{-8}\left[\mathrm{~N} / \mathrm{m}\right.$ ] [9] while others insist $\Sigma \simeq 1 \times 10^{-8}[\mathrm{~N} / \mathrm{m}$ ] [10]. For a microemulsion droplet of size $r_{0} \simeq 5 \times 10^{-9}[\mathrm{~m}]$, the measured bending rigidities are $\kappa \simeq$ $1 \times 10^{-20}[\mathrm{~J}]$ by SANS technique [16-18] or $\kappa \simeq 1 \times 10^{-21}[\mathrm{~J}]$ by dynamic Kerr effect measurement [19] yielding $\kappa / r_{0}^{2} \simeq 4 \times 10^{-4}$ or $10^{-5}[\mathrm{~N} / \mathrm{m}$ ], respectively. (See Table 1.) These values can be compared with the relatively small two-dimensional pressure 
Table 1

Orders of various hydrodynamical time scales. We used $v_{c} \simeq 10^{3}[\mathrm{~m} / \mathrm{s}], \rho \simeq 10^{3}\left[\mathrm{~kg} / \mathrm{m}^{3}\right]$ and $\eta \simeq 10^{-3}$ $\left[\mathrm{Ns} / \mathrm{m}^{2}\right]$

\begin{tabular}{lll}
\hline & Vesicle & Microemulsion droplet \\
\hline$r_{0}[\mathrm{~m}]$ & $10^{-5}$ & $10^{-8}$ \\
$B[\mathrm{~N} / \mathrm{m}]$ & $10^{-1}$ & $10^{-2} *$ \\
$\kappa[\mathrm{J}]$ & $10^{-19}$ & $10^{-20}$ \\
$\kappa / r_{0}^{2}[\mathrm{~N} / \mathrm{m}]$ & $10^{-9}$ & $10^{-4}$ \\
$\omega_{C}[1 / \mathrm{s}]$ & $10^{8}$ & $10^{11}$ \\
$1 / \tau_{\eta}[1 / \mathrm{s}]$ & $10^{4}$ & $10^{10}$ \\
$\omega_{B}[1 / \mathrm{s}]$ & $10^{6}$ & $10^{10}$ \\
$1 / \tau_{B}[1 / \mathrm{s}]$ & $10^{7}$ & $10^{9}$ \\
$\omega_{s}[1 / \mathrm{s}]$ & $10^{6}$ & $10^{9}$ \\
$\omega_{\kappa}[1 / \mathrm{s}]$ & $10^{2}$ & $10^{9}$ \\
$1 / \tau_{\kappa}[1 / \mathrm{s}]$ & $10^{-1}$ & $10^{7}$ \\
\hline
\end{tabular}

* See the text.

$7 \times 10^{-5}[\mathrm{~N} / \mathrm{m}][17]$, although they claim that it is different from the macroscopic surface tension.

In either case, it is somewhat delicate to ignore the apparent tension $\Sigma\left(\rho_{50}\right)$ in Eq. (4.10). For the simplicity of the present argument, however, we assume here the tensionless case, i.e., $\Sigma\left(\rho_{s 0}\right) \simeq 0$. In order to extract the property due to the bending rigidity in an emphasized way, it is recommended to prepare droplets of small size.

As we have done in Sections 4 and 5, several limiting cases of the effective viscosity have been considered according to the relation between $B$ and $S \approx \kappa / r_{0}^{2}$. The question is to which case the real system corresponds. By a mechanical experiment, Evans and coworkers found $B \simeq 0.14\left[\mathrm{Nm}^{-1}\right]$ for egg lecithin bilayers [70-72]. Therefore for a vesicle, we have typically $B / S \sim B r_{0}^{2} / K \simeq 1.4 \times 10^{8}$ and the membrane can be considered to be almost incompressible. It should be noticed that even $S \sim \kappa / r_{0}^{2}$ is negligibly small compared to $B$, this does not mean $S=0$ which corresponds to the different limiting case.

Another possible argument may be given as follows. Let us regard the membrane as a homogeneous elastic shell with a thickness $a$. According to the shell theory $[29,69,73]$, two-dimensional compression modulus $B$ and the bending rigidity $\kappa$ are given by

$$
B=\frac{Y a}{2(1-\sigma)} \quad \text { and } \quad \kappa=\frac{Y a^{3}}{12\left(1-\sigma^{2}\right)},
$$

respectively, where $Y$ and $\sigma$ are Young's modulus and Poisson ratio for an isotropic material in three dimensions. Then one can see that $B / S$ is approximately determined by $B / S \approx\left(r_{0} / a\right)^{2}$ [11]. For a bilayer membrane, Evans took into account the fact that membrane is not a continuum in the thickness direction and obtained different thickness dependence of the bending rigidity as $[72,74,75]$

$$
\kappa=B a^{2} / 2 .
$$


In this case we also have the same scaling behavior as $B / S \approx\left(r_{0} / a\right)^{2}$. For a lipid vesicle of size $r_{0} \simeq 10^{-5}[\mathrm{~m}]$ and $a \simeq 4 \times 10^{-9}[\mathrm{~m}][11,29]$, one finds $B / S \simeq 6 \times 10^{6}$ corresponding to the incompressible case $B \gg S \neq 0$ as before. For a microemulsion droplet, however, since the size and thickness are typically $r_{0} \simeq 5 \times 10^{-9}[\mathrm{~m}]$ and $a \simeq 2 \times 10^{-9}[\mathrm{~m}], B$ and $S$ take rather comparable values. Thus we cannot simply employ the incompressible condition for the interface of microemulsion droplets and it might be better to take both $B$ and $\kappa$ into account in the consideration of the effective viscosity.

There are several time scales involved in the present problem; each of them reflecting the corresponding mode coupled to the ambient fluids. We consider now whether these time scales can be well separated from each other. As mentioned above, we ignore the time scales which come from the surface tension $\Sigma$. In Section 3.1, we used the stationary Stokes equation Eq. (3.13) to describe the creeping motion of the surrounding fluids. This can be justified as long as the Reynolds number of the fluids $\mathrm{R}=\rho v r_{0} / \eta$ is sufficiently small ( $\rho$ is the fluid mass density). For a given frequency $\omega$, Reynolds number can be given in terms of the viscous diffusion rate $1 / \tau_{\eta} \sim \eta / \rho r_{0}^{2}$ as $\mathrm{R} \sim \omega \tau_{\eta}$. Hence the above condition sets the upper boundary value of the allowed frequency range such that $\omega \ll 1 / \tau_{\eta}$. On the other hand, the incompressible condition of the fluids Eq. (3.2) requires $\omega \ll \omega_{c} \sim v_{c} / r_{0}$ where $v_{c}$ is the speed of sound.

For the modes related to the compression modulus $B$, we have the oscillating mode $\omega_{B} \sim\left(B / \rho r_{0}^{3}\right)^{1 / 2}$ and the overdamped decaying mode (or Lucassen mode [76-78]) $1 / \tau_{B} \sim B / \eta r_{0}$. Moreover there exists an additional surface mode connected with the redistribution of $\rho_{s}$ for compressible membranes. This mode is known to have an unusual dispersion law $[23,24,38,46,47]$ :

$$
\omega_{s}=\frac{\sqrt{3}-i}{2}\left(\frac{B^{2}}{\rho \eta r_{0}^{4}}\right)^{1 / 3} .
$$

For the bending modes, on the other hand, we have the oscillating mode $\omega_{\kappa} \sim$ $\left(\kappa / \rho r_{0}^{5}\right)^{1 / 2}$ and the overdamped mode $1 / \tau_{\kappa} \sim \kappa / \eta r_{0}^{3}$. Notice that Eq. (6.3) is derived in the limit of $\omega \gg 1 / \tau_{\eta}, \omega_{\kappa}$ and may be irrelevant to the viscosity measurement. Order estimations of these time scales both for vesicles and microemulsion droplets are listed in Table 1. (Since there is no published experimental value of $B$ for microemulsion droplets, as far as we know, it is roughly estimated by $B \sim \kappa / a^{2}$ with $a \simeq 10^{-9}[\mathrm{~m}]$.) For the estimation of these quantities, we used $v_{c} \simeq 10^{3}[\mathrm{~m} / \mathrm{s}], \eta \simeq 10^{-3}\left[\mathrm{Ns} / \mathrm{m}^{2}\right]$ and $\rho \simeq 10^{3}\left[\mathrm{~kg} / \mathrm{m}^{3}\right]$.

For vesicles, bending modes are observable since $1 / \tau_{\kappa} \ll 1 / \tau_{\eta}$, while incompressible condition for the membrane is regarded to be reasonable since $\omega_{B}, 1 / \tau_{B}, \omega_{s} \gg 1 / \tau_{\eta}$. It can be also seen that the overdamped bending mode $1 / \tau_{\kappa}$ is well separated from oscillating bending mode $\omega_{\kappa}$. In the microemulsion droplet case, although the allowed frequency range is rather high, $\omega \ll 1 / \tau_{\eta} \simeq 10^{10}[1 / \mathrm{s}]$, several modes are in the same order close to $1 / \tau_{\eta}$ and might be difficult to separate from each other. Moreover these frequencies lie near the upper limit frequency which is measurable by mechanical 
experiments. The longest relaxation time $1 / \tau_{\kappa} \simeq 10^{7}[1 / \mathrm{s}]$ turns out be quite large compared to the vesicles and becomes rather comparable to $1 / \tau_{B} \simeq 10^{9}[1 / \mathrm{s}]$. Hence both of the modes should be taken into account in this case as mentioned before.

\section{Acknowledgements}

We would like to thank Prof. K. Kitahara and Prof. A. Onuki for their interest and useful comments. Fruitful interactions with Dr. Y. Fujitani, Dr. H. Kodama and Miss T. Ayako are also acknowledged.

\section{Appendix A. Derivation of the membrane force}

In this appendix, we briefly give the derivation of the restoring force due to the membrane Eq. (2.14) following mainly the calculation by Zhong-can and Helfrich [37]. In the present case, $\sigma\left(\rho_{s}\right)$ in Eq. (2.11) is no more a Lagrange multiplier and depends on $\rho_{s}$. We introduce a virtual displacement denoted by $\varepsilon\left(s^{1}, s^{2}\right)$ which is the difference between a point on the actual surface and some point on the neighboring varied surface $\hat{\boldsymbol{r}}$,

$$
\hat{r}=\boldsymbol{r}+\varepsilon n .
$$

Notice that $r$ describes not only the undeformed surface but also the deformed shape and $\varepsilon$ can be different from the actual displacement $\ell$ introduced in Eq. (2.26). Let us use $\hat{g}_{i j}, \hat{h}_{i j}, \hat{g}$ and $\hat{H}$ for the metric tensor, curvature tensor, determinant of the metric tensor and mean curvature of the varied surface, respectively. The tangent vector of the varied surface is

$$
\hat{\boldsymbol{r}}_{i}=\boldsymbol{r}_{\boldsymbol{i}}+\varepsilon_{i} \boldsymbol{n}+\varepsilon \boldsymbol{n}_{\boldsymbol{i}},
$$

with which we find

$$
\hat{g}_{i j} \approx g_{i j}-2 \varepsilon h_{i j} .
$$

Hence

$$
\hat{g} \approx g(1-4 \varepsilon H) .
$$

The local areal change is obviously

$$
\sqrt{\hat{g}} \approx \sqrt{g}(1-2 \varepsilon H) .
$$

In addition, we have

$$
\begin{aligned}
& \hat{g}^{i j} \approx g^{i j}+2 \varepsilon\left(2 H g^{i j}-K h^{i j}\right), \\
& \hat{h}_{i j} \approx h_{i j}+\varepsilon_{i j}+\varepsilon\left(K g_{i j}-2 H h_{i j}\right)-\varepsilon_{k} \Gamma_{i j}^{k},
\end{aligned}
$$


where the Christoffel symbols $\Gamma_{i j}^{k}$ are defined in Eq. (2.8). In the above, we have used the Weingarten equation Eq. (2.9) and the relation

$$
h_{i j} g^{j k} h_{k l}=2 H h_{i l}-K g_{i l}
$$

From Eqs. (2.5), (A.6) and (A.7), one can obtain the mean curvature of the varied surface as

$$
\hat{H} \approx H+\varepsilon\left(2 H^{2}-K\right)+\frac{1}{2} g^{i j} D_{i} \varepsilon_{j}
$$

Next the first variation of $H_{f}(\mathrm{Eq} .(2.11))$ is given by

$$
\delta H_{f}=\delta H_{b}+\oint_{A_{0}} \delta \sigma\left(\rho_{s}\right) \mathrm{d} A+\oint_{A_{0}} \sigma\left(\rho_{s}\right) \delta \mathrm{d} A
$$

where

$$
\begin{aligned}
\delta H_{b} & =\frac{1}{2} \kappa \delta \oint_{A_{0}}\left(2 H+c_{0}\right)^{2} \mathrm{~d} A \\
& =\frac{1}{2} \kappa \oint_{A_{0}}\left[\left(2 H+c_{0}\right)^{2} \delta \mathrm{d} A+4\left(2 H+c_{0}\right)(\delta H) \mathrm{d} A\right]
\end{aligned}
$$

According to Eq. (2.13), the second term on the r.h.s. of Eq. (A.10) becomes

$$
\oint_{A_{0}} \delta \sigma\left(\rho_{s}\right) \mathrm{d} A=\oint_{A_{0}} \frac{\partial \sigma \rho_{s}}{\partial \rho_{s}}\left(\delta \rho_{s}\right) \mathrm{d} A=-\oint_{A_{0}} \rho_{s} \frac{\partial \sigma\left(\rho_{s}\right)}{\partial \rho_{s}} \delta \mathrm{d} A
$$

Using that the first variation of $A$ is

$$
\delta \oint_{A_{0}} \mathrm{~d} A=-\oint_{A_{0}} 2 \varepsilon H \mathrm{~d} A
$$

and after the integration of $\varepsilon_{i j}$ and $\varepsilon_{k}$ by parts, we obtain

$$
\delta H_{f}=\oint_{A_{0}} \varepsilon\left[-2 \Sigma\left(\rho_{s}\right) H+\kappa\left(2 H+c_{0}\right)\left(2 H^{2}-2 K-c_{0} H\right)+2 \kappa \nabla_{L B}^{2} H\right] \mathrm{d} A
$$

where $\Sigma\left(\rho_{s}\right)$ and $\nabla_{L B}^{2}$ are defined by Eqs. (2.15) and (2.16), respectively. Finally the restoring force due to the membrane is calculated by

$$
F_{\|}=-\frac{\delta H_{f}}{\delta \varepsilon}
$$

which simply leads to Eq. (2.14). 


\section{Appendix B. Definition of the divergence operator}

In this appendix, we give the mathematical definition of the divergence operator appearing in Eq. (2.22) in the general geometry [53]. First we raise the indices by

$$
\boldsymbol{r}^{i}=g^{i j} \boldsymbol{r}_{j}
$$

with which we define

$$
v_{s}^{i}=\boldsymbol{v}_{s} \cdot \boldsymbol{r}^{i}
$$

Then the two-dimensional divergence stands for

$$
\operatorname{div}_{\perp}\left(\rho_{s} v_{s}\right)=\frac{1}{\sqrt{g}} \partial_{i}\left(\sqrt{g} \rho_{s} v_{s}^{i}\right)
$$

\section{Appendix C. Derivation of (3.30)}

In this appendix, we show the derivation of Eq. (3.30) by following the paper by Schowalter, Chaffey and Brenner [59]. Using Eqs. (3.28) and (3.29) the stress tensor Eq. (3.8) can be written as

$$
\left\langle\Pi_{\alpha \beta}\right\rangle=\eta\left(\frac{\partial v_{\alpha}^{\infty}}{\partial x_{\beta}}+\frac{\partial v_{\beta}^{\infty}}{\partial x_{\alpha}}\right)+J_{\alpha \beta}
$$

We can obtain $\eta^{*}$ defined in Eq. (3.31) by evaluating $J_{\alpha \beta}$. By substituting Eq. (3.26) into Eq. (3.28), we have

$$
\boldsymbol{\sigma}^{\infty}=2 \eta \Gamma\left(2 Y_{20}(\theta, \varphi) e_{r} e_{r}+\frac{\partial Y_{20}}{\partial \theta} e_{r} e_{\theta}\right) e^{i \omega t}
$$

The viscous stress tensor of the unperturbed flow is expressed in terms of the spherical harmonics $Y_{20}(\theta, \varphi)$. We shall find $J_{\alpha \beta}$ having this symmetry. Since the integral in Eq. (3.29) can be taken over a large closed surface $A$ lying entirely in the ambient fluid enclosing the droplet but no other ones, only the terms of order $1 / r^{2}$ are relevant in the velocity field Eq. (3.23). Thus it is sufficient to consider only

$$
v(r, t)-v^{\infty}(r, t)=\frac{1}{2 \eta} Q r
$$

where

$$
Q=P_{20}\left(\frac{r_{0}}{r}\right)^{3} Y_{20}(\theta, \varphi) e^{i \omega t}
$$

Then we can readily see that

$$
\Pi_{\alpha \beta}-\Pi_{\alpha \beta}^{\infty}=\frac{1}{2}\left(x_{\alpha} \frac{\partial Q}{\partial x_{\beta}}+x_{\beta} \frac{\partial Q}{\partial x_{\alpha}}\right) .
$$


Substituting Eqs. (C.5) and (C.3) into Eq. (3.29), one obtains

$$
J_{\alpha \beta}=c\left(\frac{1}{2} \int \frac{\partial Q}{\partial x_{\alpha}} x_{\beta} r^{3} \mathrm{~d} \Omega+\frac{1}{2} \int x_{\alpha} x_{\beta} x_{\gamma} \frac{\partial Q}{\partial x_{\gamma}} r \mathrm{~d} \Omega-\int x_{\alpha} x_{\beta} Q r \mathrm{~d} \Omega\right),
$$

where $\mathrm{d} \Omega=\sin \theta \mathrm{d} \theta \mathrm{d} \varphi$ is an element of area on a unit sphere. With the aid of Euler's theorem for homogeneous polynomials,

$$
x_{\alpha} \frac{\partial Q}{\partial x_{\alpha}}=r \frac{\partial Q}{\partial r}=-3 Q
$$

Eq. (C.6) can be simplified to

$$
J_{\alpha \beta}=c\left(-\frac{5}{2} \int x_{\alpha} x_{\beta} Q r \mathrm{~d} \Omega+\frac{1}{2} \int \frac{\partial Q}{\partial x_{\alpha}} x_{\beta} r^{3} \mathrm{~d} \Omega\right) .
$$

To evaluate Eq. (C.8) we note that

$$
\begin{aligned}
\operatorname{grad} Q= & -P_{20} \frac{r_{0}^{3}}{r^{4}}\left(\frac{1}{2}\left[Y_{31}(\theta, \varphi)+Y_{3,-1}(\theta, \varphi)\right] \boldsymbol{e}_{x}\right. \\
& \left.+\frac{1}{2 i}\left[Y_{31}(\theta, \varphi)-Y_{3,-1}(\theta, \varphi)\right] \boldsymbol{e}_{y}+3 Y_{30}(\theta, \varphi) \boldsymbol{e}_{z}\right) e^{i \omega t},
\end{aligned}
$$

and

$$
\boldsymbol{e}_{r}=\left(\begin{array}{c}
{\left[Y_{11}(\theta, \varphi)+Y_{1,-1}(\theta, \varphi)\right] / 2} \\
{\left[Y_{11}(\theta, \varphi)-Y_{1,-1}(\theta, \varphi)\right] / 2 i} \\
Y_{10}(\theta, \varphi)
\end{array}\right)
$$

Owing to the orthogonality of the spherical harmonics, only the first term remains in Eq. (C.8), hence

$$
\boldsymbol{J}=-\frac{5}{2} c P_{20} r_{0}^{3}\left(\int Y_{20}(\theta, \varphi) \boldsymbol{e}_{r} \boldsymbol{e}_{r} \mathrm{~d} \Omega\right) e^{i \omega t}
$$

From the explicit expression $Y_{20}=\left(3 \cos ^{2} \theta-1\right) / 2$, it can be seen that the variable $\varphi$ appears in Eq. (C.11) only through $e_{r}$. By integrating over $\varphi$, one finds

$$
\begin{aligned}
J= & -\frac{5 \pi}{2} c P_{20} r_{0}^{3}\left(\int_{0}^{\pi} \sin ^{3} \theta Y_{20}(\theta, \varphi) \mathrm{d} \theta\left[e_{x} e_{x}+e_{y} e_{y}\right]\right. \\
& \left.+2 \int_{0}^{\pi} \sin \theta \cos ^{2} \theta Y_{20}(\theta, \varphi) \mathrm{d} \theta e_{z} e_{z}\right) e^{i \omega t}
\end{aligned}
$$

Evaluating the integrals as

$$
\int_{0}^{\pi} \sin ^{3} \theta Y_{20}(\theta, \varphi) \mathrm{d} \theta=-\int_{0}^{\pi} \sin \theta \cos ^{2} \theta Y_{20}(\theta, \varphi) \mathrm{d} \theta=-\frac{4}{15},
$$


we finally obtain

$$
J=-\frac{1}{2} P_{20} \phi\left(2 Y_{20}(\theta, \varphi) e_{r} e_{r}+\frac{\partial Y_{20}}{\partial \theta} e_{r} e_{\theta}\right) e^{i \omega t}
$$

where $\phi=(4 \pi / 3) c r_{0}^{3}$. Comparing Eq. (C.14) with Eq. (C.2), we obtain Eq. (3.30).

\section{Appendix D. Relaxation times}

In this appendix, we list the sequence of the relaxation time $\tau_{n m}$ associated with the membrane deformation in accordance with the various limiting cases discussed in the text. Given $\tau_{n m}$, the time correlation function of the out-of-plane displacement $\left\langle\ell_{n m}(t) \ell_{n m}(0)^{*}\right\rangle$ is found to be proportional to $\exp \left(-t / \tau_{n m}\right)$. In order to calculate $\tau_{n m}$, we let $v^{\infty}(\boldsymbol{r}, t)=0$, i.e., no external flow. In such a situation, the time dependent flow fields inside and outside of the droplet are purely generated by the shape fluctuation of the membrane. Combining Eqs. (4.2), (4.3), (4.8) and (4.9) with $\Gamma=0$, one has 5 homogeneous equations for 5 unknowns $P_{n m}^{\prime}, \psi_{n m}^{\prime}, P_{n m}, \psi_{n m}$ and $\ell_{n m}$. The nontrivial solutions can be found when $\tau_{n m}=-i \omega$ satisfies the following quadratic equation for each set of $(n, m)[27]$ :

$$
A_{n m}\left(1 / \tau_{n m}\right)^{2}-B_{n m}\left(1 / \tau_{n m}\right)+C_{n m}=0
$$

where

$$
\begin{aligned}
A_{n m}= & {\left[2(n-1)(n+1) E+2 n^{2}+1\right]\left[\left(2 n^{2}+4 n+3\right) E+2 n(n+2)\right], } \\
B_{n m}= & \frac{S_{n m}}{\eta r_{0}}(n-1) n(n+1)(n+2)(2 n+1)(E+1) \\
& +\frac{B}{\eta r_{0}} n(n+1)\left[(n-1)\left(2 n^{2}+5 n+5\right) E+(n+2)\left(2 n^{2}-n+2\right)\right], \\
C_{n m}= & \frac{B S_{n m}}{\eta^{2} r_{0}^{2}}(n-1) n^{2}(n+1)^{2}(n+2),
\end{aligned}
$$

with

$$
S_{n m}=\Sigma\left(\rho_{s 0}\right)+\frac{\kappa}{r_{0}^{2}}\left[n(n+1)-2 c_{0} r_{0}+\frac{1}{2} c_{0}^{2} r_{0}^{2}\right]
$$

For $n=2$, Eq. (D.1) reduces to the equation which is obtained by putting the denominator of Eq. (4.15) equal to zero. We list below the limiting expressions of $\tau_{n m}$ according to the relation between $B$ and $S_{n m}$.

(i) $B \gg S_{n m}>0$

$$
\frac{1}{\tau_{n m}}=\frac{S_{n m}}{\eta r_{0}} \frac{(n-1) n(n+1)(n+2)}{(n-1)\left(2 n^{2}+5 n+5\right) E+(n+2)\left(2 n^{2}-n+2\right)} .
$$


(ii) $S_{n m} \gg B>0$

$$
\frac{1}{\tau_{n n l}}=\frac{B}{\eta r_{0}} \frac{n(n+1)}{(2 n+1)(E+1)} \text {. }
$$

(iii) $S_{n m}>B=0$

$$
\begin{aligned}
\frac{1}{\tau_{n n}}= & \frac{S_{n m}}{\eta r_{0}} \\
& \times \frac{(n-1) n(n+1)(n+2)(2 n+1)(E+1)}{\left[2(n-1)(n+1) E+2 n^{2}+1\right]\left[\left(2 n^{2}+4 n+3\right) E+2 n(n+2)\right]} .
\end{aligned}
$$

(iv) $B>S_{n m}=0$

$$
\begin{aligned}
\frac{1}{\tau_{n m}}= & \frac{B}{\eta r_{0}} \\
& \times \frac{n(n+1)\left[(n-1)\left(2 n^{2}+5 n+5\right) E+(n+2)\left(2 n^{2}-n+2\right)\right]}{\left[2(n-1)(n+1) E+2 n^{2}+1\right]\left[\left(2 n^{2}+4 n+3\right) E+2 n(n+2)\right]} .
\end{aligned}
$$

For $n=2$, Eqs. (D.6), (D.7), (D.8) and (D.9) give Eqs. (4.19), (4.23), (4.26) and (4.30), respectively. Eq. (D.6) was first derived in Refs. [5,20] for $E=1$ and a more general case was obtained recently in Refs. [26,27] while Eq. (D.8) was calculated in Refs. [22,24].

\section{References}

11] R. Lipowsky, D. Richter and E. Sackmann, eds., Structure and Conformation of Amphiphilic Membranes (Springer, Berlin, 1992).

[2] D. Beysens, N. Boccara and G. Forgacs, eds., Dynamical Phenomena at Interfaces, Surfaces and Membranes (Nova, New York, 1993).

13] K.L. Mittal and B. Lindman, eds., Surfactants in Solution (Plenum, New York, 1984).

[4] W. Helfrich, Z. Naturforsch. 28c (1973) 693.

[5] M.B. Schneider, J.T. Jenkins and W.W. Webb, J. Phys. (Paris) 45 (1984) 1457.

[6] H. Engelhardt, H.P. Duwe and E. Sackmann, J. Phys. Lett. (Paris) 46 (1985) L395.

17] H.P. Duwe, H. Engelhardt, A. Zilker and E. Sackmann, Mol. Cryst. Liq. Cryst. 152 (1987) 1.

[8] I. Bivas, P. Hanusse, P. Bothorel, J. Lalanne and O. Aguerre-Chariol, J. Phys. (Paris) 48 (1987) 855.

[9] J.F. Faucon, M.D. Mitov, P. Méléard, I. Bivas and P. Bothorel, J. Phys. (Paris) 50 (1989) 2389.

[10] P. Méléard, M.D. Mitov, J.F. Faucon and P. Bothorel, Europhys. Lett. 11 (1990) 355.

[11] M. Mutz and W. Helfrich, J. Phys. (Paris) 51 (1990) 991.

[12] H.P. Duwe, J. Kaes and E. Sackmann, J. Phys. (Paris) 51 (1990) 945.

[13] A. Zilker, H. Engelhardt and E. Sackmann, J. Phys. Lett. (Paris) 48 (1987) 2139.

[14] A. Zilker, M. Ziegler and E. Sackmann, Phys. Rev. A 46 (1992) 7998.

[15] M.A. Peterson, H. Strey and E. Sackmann, J. Phys. II (Paris) 2 (1992) 1273.

[16] S. Huang, S.T. Milner, B. Farago and D. Richter, Phys. Rev. Lett. 59 (1987) 2600.

[17] B. Farago, D. Richter, S. Huang, S.A. Safran and S.T. Milner, Phys. Rev. Lett. 65 (1990) 3348. 
[18] B. Farago, S. Huang, D. Richter, S.A. Safran and S.T. Milner, Prog. Colloid Polymer Sci. 81 (1990) 60.

[19] E. v.d. Linden, D. Bedeaux, R. Hilfiker and H.F. Eicke, Ber. Bunsenges. Phys. Chem. 95 (1991) 876.

[20] S.T. Milner and S.A. Safran, Phys. Rev. A 36 (1987) 4371.

[21] E. v.d. Linden, D. Bedeaux and M. Borkovec, Physica A 162 (1989) 99.

[22] S. Komura and K. Seki, Physica A 192 (1993) 27.

[23] V.V. Lebedev and A.R. Muratov, Sov. Phys. JETP 68 (1989) 1011.

[24] V. Lisy, Phys. Lett. A 150 (1990) 105; 152 ( I991) 504.

[25] V. Lisy, A.V. Zatovsky and A.V. Zvelindovsky, Physica A 183 (1992) 262.

[26] A. Onuki, Europhys. Lett. 24 (1993) 151.

[27] Y. Fujitani, Physica A 203 (1994) 214.

[28] J. Mellema, C. Blom and J. Beekwilder, Rheologica Acta 26418 (1987).

[29] J.B.A.F. Smeulders, C. Blom and J. Mellema, Phys. Rev. A 42 (1990) 3843.

[30] J.B.A.F. Smeulders, J. Mellema and C. Blom, Phys. Rev. A 46 (1992) 7708.

[31] J.G. Oldroyd, Proc. R. Soc. London A 218 (1953) 122.

[32] J.G. Oldroyd, Proc. R. Soc. London A 232 (1955) 567.

[33] Y. Marathe and S. Ramaswamy, Europhys. Lett. 8 (1989) 581.

[34] P. Snarbe and G. Porte, Europhys. Lett. 13 (1990) 641.

[35] G.I. Taylor, Proc. R. Soc. London A 138 (1932) 41.

[36] G.I. Taylor, Proc. R. Soc. London A 146 (1934) 501.

[37] O.-Y. Zhong-can and W. Helfrich, Phys. Rev. Lett. 59 (1987) 2486; Phys. Rev. A 39 (1989) 5280.

[38] A. Onuki, J. Phys. Soc. Jpn. 62 (1993) 385.

[39] A. Einstein, Ann. Phys. 19 (1906) 289; 34 (1911) 591.

[40] H. Frölich and R. Sack, Proc. R. Soc. London A 185 (1946) 415.

[41] S.J. Choi and W.R. Schowalter, Phys. Fluids 18 (1974) 420.

[42] M. Oosterbroek and J. Mellema, J. Colloid Interface Sci. 84 (1981) 14.

[43] A. Onuki and K. Kawasaki, Europhys. Lett. 18 (1992) 729.

[44] F. Brochard, P.G. de Gennes and P. Pfeuty, J. Phys. (Paris) 37 (1976) 1099.

[45] P.G. de Gennes and C. Taupin, J. Phys. Chem. 86 (1982) 2294.

[46] E.I. Kats and V.V. Lebedev, Sov. Phys. JETP 67 (1988) 940.

[47] V.V. Lebedev, Phys. Scr. T29 (1989) 255.

[48] W. Helfrich and R.M. Servuss, Nuovo Cimento D 3 (1984) 137.

[49] Y. Marathe and S. Ramaswamy, J. Phys. (Paris) 51 (1990) 2143.

[50] E.I. Kats and V.V. Lebedev, Europhys. Lett. 22 (1993) 469.

[51] W. Helfrich and M.M. Kozlof, J. Phys. II (Paris) 3 (1993) 287.

[52] L.D. Landau and E.M. Lifshitz, Fluid Mechanics (Pergamon, Oxford, 1970).

[53] R. Aris, Vectors, Tensors and Basic Equations of Fluid Mechanics (Dover, New York, 1989).

[54] G.K. Batchelor, J. Fluid Mech. 41 (1970) 545.

[55] J. Mellema and M.W.M. Willemse, Physica A 122 (1983) 286.

[56] C.E. Rosenkilde, J. Math. Phys. 8 (1967) 84.

[57] H. Lamb, Hydrodynamics (Cambridge Univ. Press, London, 1975).

[58] J. Happel and H. Brenner, Low Reynolds Number Hydrodynamics (Prentice-Hall, Eaglewood Cliffs, NJ, 1965).

[59] W.R. Schowalter, C.E. Chaffey and H. Brenner, J. Colloid Interface Sci. 26 (1968) 152.

[60] M. Schwartz and S.F. Edwards, Physica A 153 (1988) 355.

[61] M. Schwartz and S.F. Edwards, Physica A 167 (1990) 589.

[62] V. Levich, Physicochemical Hydrodynamics (Prentice-Hall, Eaglewood Cliffs, NJ, 1970).

[63] R. Simha, J. Appl. Phys. 23 (1952) 1020.

[64] J. Happel, J. Appl. Phys. 28 (1957) 1288.

[65] T. Miloh and Y. Benveniste, Phys. Fluids A 1 (1989) 1915.

[66] D.G. Thomas, J. Colloid Sci. 20 (1965) 267.

[67] J.B. Keller, L.A. Rubenfeld and J.E. Molyneux, J. Fluid Mech. 30 (1967) 97.

[68] I. Yaron and B. Gal-Or, Rheol. Acta 11 (1972) 241.

[69] L.D. Landau and E.M. Lifshitz, Theory of Elasticity (Pergamon, Oxford, 1970).

[70] R. Kwok and E. Evans, Biophys. J. 35 (1981) 637.

[71] E. Evans and D. Needham, J. Phys. Chem. 91 (1987) 4219. 
[72] E. Evans and W. Rawicz, Phys. Rev. Lett. 64 (1990) 2094.

[73] F.I. Niordson, Shell Theory (North-Holland, New York, 1985).

[74] E. Evans, Biophys. J. 14 (1974) 923.

[75] T.M. Fisher, Biophys. J. 63 (1992) 1328; J. Phys. II (Paris) 2 (1992) 327, 337; 3 (1993) 1795.

|76| J. Lucassen, Trans. Faraday Soc. 64 (1968) 2221.

[77] J. Lucassen and R.S. Hansen, J. Colloid Interface Sci. 22 (1966) 32; 23 (1967) 319

|78| L. Kramer, J. Chem. Phys. 55 (1971) 2097. 